LPENSL-TH-02/11

DESY $10-254$

\title{
Correlation functions of one-dimensional bosons at low temperature
}

\author{
K. K. Kozlowski1, J. M. Maillet2, N. A. Slavnov ${ }^{3}$
}

\begin{abstract}
We consider the low-temperature limit of the long-distance asymptotic behavior of the finite temperature density-density correlation function in the onedimensional Bose gas derived recently in the algebraic Bethe Ansatz framework. Our results confirm the predictions based on the Luttinger liquid and conformal field theory approaches. We also demonstrate that the amplitudes arising in this asymptotic expansion at low-temperature coincide with the amplitudes associated with the so-called critical form factors.
\end{abstract}

\section{Introduction}

The model of one-dimensional bosons interacting through a two-body $\delta$-function potential is described by the Quantum Nonlinear Schrödinger equation (QNLS model). Recently [1], the Lagrange series method was applied in the framework of the algebraic Bethe ansatz to the derivation of the long-distance asymptotic expansion of the finite temperature density-density

\footnotetext{
${ }^{1}$ DESY, Hamburg, Deutschland, karol.kajetan.kozlowski@desy.de

${ }^{2}$ Laboratoire de Physique, UMR 5672 du CNRS, ENS Lyon, France, maillet@ens-lyon.fr

${ }^{3}$ Steklov Mathematical Institute, Moscow, Russia, nslavnov@mi.ras.ru
} 
correlation function in this model. In the present paper, we consider the low-temperature limit of this result.

The QNLS model is given by the Hamiltonian

$$
H=\int_{0}^{L}\left(\partial_{x} \Psi^{\dagger} \partial_{x} \Psi+c \Psi^{\dagger} \Psi^{\dagger} \Psi \Psi-h \Psi^{\dagger} \Psi\right) d x .
$$

Here $\Psi$ and $\Psi^{\dagger}$ are Bose fields possessing canonical equal-time commutation relations, $c>0$ is a coupling constant and $h>0$ the chemical potential. The results obtained in [1] are relative to the thermodynamic limit $L \rightarrow \infty$ of this model.

The density operator $j(x)=\Psi^{\dagger}(x) \Psi(x)$ defines the operator of the number of particles in the interval $[0, x]$

$$
\mathcal{Q}_{x}=\int_{0}^{x} j(z) d z
$$

The generating function for the density-density correlations reads

$$
\left\langle e^{2 \pi i \alpha \mathcal{Q}_{x}}\right\rangle_{T}=\lim _{L \rightarrow \infty} \frac{\left\langle\Omega_{T}\left|e^{2 \pi i \alpha \mathcal{Q}_{x}}\right| \Omega_{T}\right\rangle}{\left\langle\Omega_{T} \mid \Omega_{T}\right\rangle}
$$

were $T$ is the temperature, $\alpha$ a complex number and $\left|\Omega_{T}\right\rangle$ any eigenstate of $H$ that goes to the state of thermal equilibrium in the infinite volume limit. Indeed, the correlation function of densities $\langle j(x) j(0)\rangle_{T}$ can be obtained from (1.3) as

$$
\langle j(x) j(0)\rangle_{T}=-\left.\frac{1}{8 \pi^{2}} \frac{\partial^{2}}{\partial x^{2}} \frac{\partial^{2}}{\partial \alpha^{2}}\left\langle e^{2 \pi i \alpha \mathcal{Q}_{x}}\right\rangle_{T}\right|_{\alpha=0} .
$$

We have shown in [1] that the large- $x$ asymptotic expansion of the generating function (1.3) (and respectively of the two-point function (1.4)) is given in terms of solutions to a set of nonlinear integral equations closely related to ones arising in the quantum transfer matrix approach [2, 3, 4, 5, 6, 7]. Below, we solve these equations in the low-temperature limit. This computation allows us to reach two goals.

On the one hand, one can argue that the Luttinger liquid [8] and conformal field theory (CFT) approaches [9, 10, 11, 12, 13, 14, 15, 16, can be used to predict the large- $x$ asymptotic behavior of the low-temperature correlation functions in massless one dimensional quantum models. The QNLS model does belong to this class. Thus, our results give us a possibility to confirm these predictions by a direct derivation based on the algebraic Bethe ansatz. Namely, we show that in the low-temperature limit $(T \rightarrow 0)$ the asymptotic expansion $(x \rightarrow \infty, x T \rightarrow \infty)$ of the density-density correlation function takes the following form (at leading order for each oscillatory term):

$$
\langle j(x) j(0)\rangle_{T}=D^{2}-\frac{\left(T \mathcal{Z} / v_{0}\right)^{2}}{2 \sinh ^{2}\left(\pi T x / v_{0}\right)}+\sum_{\ell \in \mathbb{Z}^{*}} A_{\ell} e^{2 i x \ell k_{F}}\left(\frac{\pi T / v_{0}}{\sinh \left(\pi T x / v_{0}\right)}\right)^{2 \ell^{2} \mathcal{Z}^{2}} .
$$


Above appear several constants that will be defined in section 3, namely, the average density of the gas $D$, the Fermi momentum $k_{F}$, the velocity of sound on the Fermi boundary $v_{0}$ and the value of the dressed charge at Fermi boundary $\mathcal{Z}$. The coefficients $A_{\ell}$ do not depend on $T$. All the dependence of the amplitudes on $T$ has been gathered in the pre-factor $\left(\pi T / v_{0}\right)^{2 \ell^{2} \mathcal{Z}^{2}}$.

This form is in full agreement with the CFT predictions. Moreover it provides one with an asymptotic behavior that is also valid in the full scaling region of $x T$ and in particular in the $T=0$ case.

On the other hand our approach allows us to calculate the constant coefficients $A_{\ell}$ in (1.5). We show that $A_{\ell}$ are related to the amplitudes of the so-called critical form factors introduced in [17] and arising in the study of the model at $T=0$. More precisely, the coefficients $A_{\ell}\left(\pi T / v_{0}\right)^{2 \ell^{2} \mathcal{Z}^{2}}$ determined for the system in the thermodynamic limit and at small but finite temperature $T$ are equal to the amplitudes of the critical form factors corresponding to umklapptype excited states of momentum $2 k_{F} \ell$ and determined for the system of large but finite size $L$ at zero temperature, with the identification $v_{0} / T \mapsto i L$. We will show this coincidence by means of straightforward calculations carried in the core of this paper.

This article is organized as follows. In section 2 we recall the results obtained in [1]. In section 3 we present the thermodynamics of the QNLS model at low temperature. In particular we solve the non-linear integral equation determining the asymptotic expansion of the correlation function in the low-temperature approximation. This allows us to obtain the rates of exponential decays in section 4 and the constant amplitudes in section 5 . The expansion (1.5) is derived in section 6. Various estimates of the low-temperature behavior of the integrals that we deal with are gathered in three appendices.

\section{Long distance asymptotic behavior at general temperature}

The state of the thermal equilibrium in the QNLS model is described by the Yang-Yang equation [18] for the excitation energy $\varepsilon(\lambda)$

$$
\varepsilon(\lambda)=\lambda^{2}-h-\frac{T}{2 \pi} \int_{\mathbb{R}} K(\lambda-\mu) \log \left(1+e^{-\frac{\varepsilon(\mu)}{T}}\right) d \mu,
$$

and the integral equation for the total density $\rho_{t}(\lambda)$

$$
\rho_{t}(\lambda)-\frac{1}{2 \pi} \int_{\mathbb{R}} K(\lambda-\mu) \vartheta(\mu) \rho_{t}(\mu) d \mu=\frac{1}{2 \pi} .
$$

The kernel $K(\lambda)$ and the Fermi weight $\vartheta(\lambda)$ appearing above read

$$
K(\lambda)=\frac{2 c}{\lambda^{2}+c^{2}}, \quad \vartheta(\lambda)=\left(1+e^{\frac{\varepsilon(\lambda)}{T}}\right)^{-1} .
$$

Below the poles of the Fermi weight will play an important role. Therefore we introduce the roots $\left\{r^{ \pm}\right\}$of the equation $1+e^{-\varepsilon\left(r_{j}^{ \pm}\right) / T}=0$, where $r_{j}^{+}$(resp. $r_{j}^{-}$) belong to the upper (resp. lower) half-plane (see Fig. 1). 
The asymptotic expansion of the generating function $\left\langle e^{2 \pi i \alpha \mathcal{Q}_{x}}\right\rangle_{T}$ is given in terms of the solutions to non-linear integral equations similar to (2.1). Let us choose $n$ points $(n=0,1, \ldots)$ $\hat{s}_{j}^{+}$in the upper half-plane and $n$ points $\hat{s}_{j}^{-}$in the lower half-plane. We then introduce non-linear integral equation for a function $u(\lambda)$

$$
u(\lambda)=\lambda^{2}-h_{\alpha}-\frac{T}{2 \pi} \int_{\mathbb{R}} K(\lambda-\mu) \log \left(1+e^{-\frac{u(\mu)}{T}}\right) d \mu+i T \sum_{j=1}^{n}\left(\theta\left(\lambda-\hat{s}_{j}^{+}\right)-\theta\left(\lambda-\hat{s}_{j}^{-}\right)\right),
$$

where $h_{\alpha}=h+2 \pi i \alpha T$ and

$$
\theta(\lambda)=i \log \left(\frac{i c+\lambda}{i c-\lambda}\right), \quad \theta^{\prime}(\lambda)=K(\lambda)
$$

Below we will show that, in the low-temperature limit and for $\left\{\hat{s}^{ \pm}\right\}$fixed, the solution to the equation (2.4) always exists. Clearly, this solution depends on the parameters $\left\{\hat{s}^{ \pm}\right\}: u(\lambda)=$ $u\left(\lambda \mid\left\{\hat{s}^{+}\right\},\left\{\hat{s}^{-}\right\}\right)$. By imposing the constraints

$$
1+\exp \left(-\frac{u\left(\hat{s}_{j}^{ \pm} \mid\left\{\hat{s}^{+}\right\},\left\{\hat{s}^{-}\right\}\right)}{T}\right)=0, \quad j=1, \ldots, n,
$$

we obtain a system of equations, which fixes the sets $\left\{\hat{s}^{ \pm}\right\}_{i}$ that are relevant for the description of the long-distance asymptotics. The subscript $i$ enumerates these sets. The long-distance asymptotic expansion for the generating function $\left\langle e^{2 \pi i \alpha \mathcal{Q}_{x}}\right\rangle_{T}$ can then be organized into a sum parameterized by the functions $u_{i}(\lambda) \equiv u_{i}\left(\lambda \mid\left\{\hat{s}^{+}\right\}_{i},\left\{\hat{s}^{-}\right\}_{i}\right)$ that solve (2.4) with a corresponding set of roots $\left\{\hat{s}^{ \pm}\right\}_{i}$ :

$$
\left\langle e^{2 \pi i \alpha \mathcal{Q}_{x}}\right\rangle_{T}=\sum_{i} e^{-x p\left[u_{i}\right]} B\left[u_{i}\right]+o\left(e^{-x \max \Re\left(p\left[u_{i}\right]\right)}\right),
$$

where $p\left[u_{i}\right]$ and $B\left[u_{i}\right]$ are functionals of $u_{i}(\lambda)$ whose explicit form will be specified later.

Observe that equation (2.4) can be recast in the form

$$
u_{i}(\lambda)=\lambda^{2}-h_{\alpha}-\frac{T}{2 \pi} \int_{\hat{\mathcal{C}}_{i}} K(\lambda-\mu) \log \left(1+e^{-\frac{u_{i}(\mu)}{T}}\right) d \mu,
$$

where the contour $\hat{\mathcal{C}}_{i}$ is such that the roots $\left\{\hat{s}^{ \pm}\right\}$are located between the real axis and $\hat{\mathcal{C}}_{i}$. We also demand that the contours $\hat{\mathcal{C}}_{i}$ separate the sets $\left\{\hat{s}^{ \pm}\right\}_{i}$ from all over possible roots of the equation $1+e^{-u_{i}(\lambda) / T}=0$ and from all the roots $\left\{r^{ \pm}\right\}$(see Fig. 1). Then one can interpret the asymptotic expansion of $\left\langle e^{2 \pi i \alpha \mathcal{Q}_{x}}\right\rangle_{T}$ as being given by the sum over the different possible choices of contours $\hat{\mathcal{C}}_{i}$.

The explicit expressions for the rates of exponential decay $p\left[u_{i}\right]$ and the amplitudes $B\left[u_{i}\right]$ can be written down in terms of integrals over the contours $\hat{\mathcal{C}}_{i}$. Let us introduce an auxiliary functions $z_{i}(\lambda)$ by

$$
z_{i}(\lambda)=-\frac{1}{2 \pi i} \log \left(\frac{1+e^{-\frac{u_{i}(\lambda)}{T}}}{1+e^{-\frac{\varepsilon(\lambda)}{T}}}\right)
$$




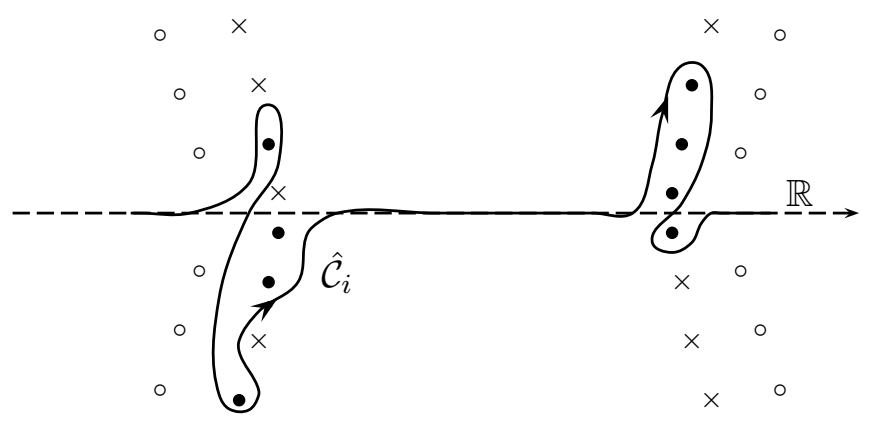

Figure 1: The roots $\left\{r^{ \pm}\right\}$are depicted by $(\circ)$, the roots $\left\{\hat{s}^{ \pm}\right\}_{i}$ are depicted by $(\bullet)$. Other roots of the equation $1+e^{-u_{i}\left(\hat{s}^{ \pm}\right) / T}=0$ are depicted by $(\times)$. The contour $\hat{\mathcal{C}}_{i}$ bypasses the roots $\left\{\hat{s}^{+}\right\}_{i}$ from above and the roots $\left\{\hat{s}^{-}\right\}_{i}$ from below. It also separates the points $\left\{\hat{s}^{ \pm}\right\}_{i}$ from the other points $\left\{\hat{s}^{ \pm}\right\}$as well as from all the poles of the Fermi weight $\left\{r^{ \pm}\right\}$.

Then the rates of exponential decays take the very simple form

$$
p\left[u_{k}\right]=i \int_{\hat{\mathcal{C}}_{k}} z_{k}(\lambda) d \lambda .
$$

The expressions for the amplitudes $B\left[u_{i}\right]$ are much more cumbersome. We present them in the form

$$
B\left[u_{i}\right]=B_{d}\left[u_{i}\right] B_{s}\left[u_{i}\right], \quad \text { where } \quad B_{d}\left[u_{i}\right]=\exp \left(\int_{\hat{\mathcal{C}}_{i}} \frac{z_{i}(\lambda) z_{i}(\mu)}{\left(\lambda-\mu_{+}\right)^{2}} d \lambda d \mu\right),
$$

and the symbol $\mu_{+}$means that the variable $\mu$ is slightly shifted to the left of the oriented integration contour $\hat{\mathcal{C}}_{i}$. The reason we separate the coefficients $B\left[u_{i}\right]$ into two factors $B_{d}\left[u_{i}\right]$ and $B_{s}\left[u_{i}\right]$ is that these go, in the low-temperature limit, to the discrete and to the smooth parts of critical form factors respectively, as defined in [17].

In order to describe $B_{s}\left[u_{i}\right]$ we first introduce the Cauchy transform operator $L_{\hat{\mathcal{C}}_{i}}$ on the contour $\hat{\mathcal{C}}_{i}$

$$
L_{\hat{\mathcal{C}}_{i}}\left[z_{i}\right](\omega)=\int_{\hat{\mathcal{C}}_{i}} \frac{z_{i}(\lambda)}{\lambda-\omega} d \lambda
$$

and a functional $C_{0}=C_{0}\left[z_{i}\right]$

$$
C_{0}\left[z_{i}\right]=\int_{\hat{\mathcal{C}}_{i}} \frac{z_{i}(\lambda) z_{i}(\mu)}{(\lambda-\mu-i c)^{2}} d \lambda d \mu
$$


Then

$$
\begin{aligned}
B_{s}\left[u_{i}\right] & =\left(e^{2 \pi i \alpha}-1\right)^{2} e^{-C_{0}\left[z_{i}\right]} \frac{\operatorname{det}\left(I+\frac{1}{2 \pi i} \hat{U}^{(1)}\left[z_{i}\right]\right) \operatorname{det}\left(I+\frac{1}{2 \pi i} \hat{U}^{(2)}\left[z_{i}\right]\right)}{\operatorname{det}\left[I-\frac{1}{2 \pi} K^{(\varepsilon)}\right] \operatorname{det}\left[I-\frac{1}{2 \pi} K^{\left(u_{i}\right)}\right]} \\
& \times\left[e^{L_{\hat{\mathcal{C}}_{i}}\left[z_{i}\right]\left(\theta_{1}+i c\right)}-e^{2 \pi i \alpha+L_{\hat{\mathcal{C}}_{i}}\left[z_{i}\right]\left(\theta_{1}-i c\right)}\right]^{-1}\left[e^{-L_{\hat{\mathcal{C}}_{i}}\left[z_{i}\right]\left(\theta_{2}-i c\right)}-e^{2 \pi i \alpha-L_{\hat{\mathcal{C}}_{i}}\left[z_{i}\right]\left(\theta_{2}+i c\right)}\right]^{-1} .
\end{aligned}
$$

The first line of (2.14) contains a ratio of Fredholm determinants. The integral operators $I-$ $\frac{1}{2 \pi} K^{(\varepsilon)}$ and $I-\frac{1}{2 \pi} K^{\left(u_{i}\right)}$ have the kernels

$$
K^{(\varepsilon)}(\lambda, \mu)=\frac{K(\lambda-\mu)}{1+e^{\frac{\varepsilon(\mu)}{T}}}, \quad K^{\left(u_{i}\right)}(\lambda, \mu)=\frac{K(\lambda-\mu)}{1+e^{\frac{u_{i}(\mu)}{T}}} .
$$

The first of these integral operators acts on the real axis and the second one acts on the contour $\hat{\mathcal{C}}_{i}$. The operators $I+\frac{1}{2 \pi i} \hat{U}^{(1)}\left[z_{i}\right]$ and $I+\frac{1}{2 \pi i} \hat{U}^{(2)}\left[z_{i}\right]$ both act on a anticlockwise oriented closed contour surrounding $\hat{\mathcal{C}}_{i}$. Their kernels are given by

$$
\hat{U}^{(1)}\left(w, w^{\prime},\left[z_{i}\right]\right)=-e^{L_{\hat{\mathcal{C}}_{i}}\left[z_{i}\right](w)} \cdot \frac{K_{\alpha}\left(w-w^{\prime}\right)-K_{\alpha}\left(\theta_{1}-w^{\prime}\right)}{e^{L_{\hat{\mathcal{C}}_{i}}\left[z_{i}\right](w+i c)}-e^{2 \pi i \alpha+L_{\hat{\mathcal{C}}_{i}}\left[z_{i}\right](w-i c)}},
$$

and

$$
\hat{U}^{(2)}\left(w, w^{\prime},\left[z_{i}\right]\right)=e^{-L_{\hat{\mathcal{C}}_{i}}\left[z_{i}\right]\left(w^{\prime}\right)} \cdot \frac{K_{\alpha}\left(w-w^{\prime}\right)-K_{\alpha}\left(w-\theta_{2}\right)}{e^{-L_{\hat{\mathcal{C}}_{i}}\left[z_{i}\right]\left(w^{\prime}-i c\right)}-e^{2 \pi i \alpha-L_{\hat{\mathcal{C}}_{i}}\left[z_{i}\right]\left(w^{\prime}+i c\right)}},
$$

where

$$
K_{\alpha}(\lambda)=\frac{1}{\lambda+i c}-\frac{e^{2 \pi i \alpha}}{\lambda-i c} .
$$

Finally, observe that the kernels $\hat{U}^{(1,2)}\left(w, w^{\prime},\left[z_{i}\right]\right)$ as well as the coefficient in the second line of (2.14) depend on arbitrary complex numbers $\theta_{1}$ and $\theta_{2}$ located inside of the contour where the operators $\hat{U}^{(1,2)}\left(w, w^{\prime},\left[z_{i}\right]\right)$ act. One can prove (see [19]) that the total combination (2.14) does not depend on the specific choice of these parameters.

\section{Thermodynamics at low temperature}

In this section we provide a list of necessary formulae describing the thermodynamics of the QNLS model at low temperature. We begin our discussion with the $T=0$ case.

\subsection{Zero temperature}

It is known [18] that for a positive chemical potential $h>0$ the solution $\varepsilon(\lambda)$ to the equation (2.1) has two roots $\pm q(T)$ on the real axis: $\varepsilon( \pm q(T))=0$. Hereby $\varepsilon(\lambda)>0$ for $|\lambda|>q(T)$ and 
$\varepsilon(\lambda)<0$ for $|\lambda|<q(T)$. Let $\varepsilon(\lambda) \rightarrow \varepsilon_{0}(\lambda)$ and $q(T) \rightarrow q$ as $T \rightarrow 0$. Then,

$$
\lim _{T \rightarrow 0} T \log \left(1+e^{-\frac{\varepsilon(\lambda)}{T}}\right)=\left\{\begin{array}{cc}
0, & |\lambda|>q, \\
-\varepsilon_{0}(\lambda), & |\lambda|<q,
\end{array} .\right.
$$

It is then straightforward to check that equation (2.1) turns into a linear integral equation for the dressed energy $\varepsilon_{0}(\lambda)$ :

$$
\varepsilon_{0}(\lambda)-\frac{1}{2 \pi} \int_{-q}^{q} K(\lambda-\mu) \varepsilon_{0}(\mu) d \mu=\lambda^{2}-h, \quad \varepsilon_{0}( \pm q)=0 .
$$

At $T=0$ the state of thermal equilibrium goes to the ground state of the QNLS model. The Fermi weight $\vartheta(\lambda)(2.3)$ turns into the characteristic function of the interval $[-q, q]$. Therefore the equation (2.2) for the density takes the form

$$
\rho_{t}(\lambda)-\frac{1}{2 \pi} \int_{-q}^{q} K(\lambda-\mu) \rho_{t}(\mu) d \mu=\frac{1}{2 \pi}, \quad T=0 .
$$

Another important characteristic of the ground state is the dressed charge $Z(\lambda)$. In the QNLS model it is proportional to the density $Z(\lambda)=2 \pi \rho_{t}(\lambda)$. Below, we will use a special notation for the value of the dressed charge on the Fermi boundary $\mathcal{Z}=Z( \pm q)$. A formal expression for $\mathcal{Z}$ can be given in terms of the resolvent to the operator $I-\frac{1}{2 \pi} K$ acting on the interval $[-q, q]$

$$
\mathcal{Z}=1+\int_{-q}^{q} R(\lambda, \pm q) d \lambda
$$

where

$$
R(\lambda, \xi)-\frac{1}{2 \pi} \int_{-q}^{q} K(\lambda-\mu) R(\mu, \xi) d \mu=\frac{1}{2 \pi} K(\lambda-\xi) .
$$

Finally, we give the formulae for the constants appearing in (1.5), namely the average density $D$, the Fermi momentum $k_{F}$, and the velocity of the sound on the Fermi boundary $v_{0}$ :

$$
D=\int_{-q}^{q} \rho_{t}(\lambda) d \lambda, \quad k_{F}=\pi D, \quad v_{0}=\frac{\varepsilon_{0}^{\prime}}{\mathcal{Z}}
$$

where we denoted $\varepsilon_{0}^{\prime} \equiv \varepsilon_{0}^{\prime}(q)$. 


\subsection{The dressed energy at low temperature}

We now describe the power-law corrections to the Yang-Yang equation (2.1), which appear at low but non-zero temperature. For $T$ small enough the solution to the equation (2.1) $\varepsilon(\lambda)$ has the following expansion [20, 21, 22]:

$$
\varepsilon(\lambda)=\sum_{k=0}^{2} T^{k} \varepsilon_{k}(\lambda)+O\left(T^{3}\right) .
$$

The functions $\varepsilon_{1}(\lambda)$ and $\varepsilon_{2}(\lambda)$ can be found from the analysis of the integral in (2.1) in small vicinities of the Fermi boundaries $\pm q$. The details of this analysis are given in appendix A (see also [20, 21, 22]).

Using (A.8) and substituting the expansion (3.7) into the Yang-Yang equation we obtain

$$
\begin{aligned}
& \sum_{k=0}^{2} T^{k} \varepsilon_{k}(\lambda)-\frac{1}{2 \pi} \sum_{k=0}^{2} T^{k} \int_{-q}^{q} K(\lambda-\mu) \varepsilon_{k}(\mu) d \mu=\lambda^{2}-h \\
& \quad-\frac{T^{2} \pi}{12 \varepsilon_{0}^{\prime}}(K(\lambda-q)+K(\lambda+q))-\frac{T^{2} K(\lambda-q) \varepsilon_{1}^{2}(q)}{4 \pi \varepsilon_{0}^{\prime}}-\frac{T^{2} K(\lambda+q) \varepsilon_{1}^{2}(-q)}{4 \pi \varepsilon_{0}^{\prime}}+O\left(T^{3}\right) .
\end{aligned}
$$

What follows from this analysis is that $\varepsilon_{1}(\lambda)=0$ and

$$
\varepsilon_{2}(\lambda)=-\frac{\pi^{2}}{6 \varepsilon_{0}^{\prime}}(R(\lambda, q)+R(\lambda,-q))
$$

where $R(\lambda, \mu)$ corresponds to the resolvent defined by (3.5).

\subsection{The poles of the Fermi weight}

We now consider the low-temperature behavior of the roots $r_{k}^{ \pm}$to the equation $1+e^{-\frac{\varepsilon(\lambda)}{T}}=0$, namely the solutions to $\varepsilon(\lambda)=2 \pi i T(k+1 / 2), k \in \mathbb{Z}$. Obviously all these roots collapse to $q$ or $-q$ when $T \rightarrow 0$. Therefore setting $r_{k}^{ \pm}=q+T \tilde{r}_{k}^{ \pm}+O\left(T^{2}\right)\left(\right.$ resp. $r_{k}^{ \pm}=-q+T \tilde{r}_{k}^{ \pm}+O\left(T^{2}\right)$ ) and substituting these expansions into $\varepsilon\left(r_{k}^{ \pm}\right)=2 \pi i T(k+1 / 2)$ we find

$$
\begin{aligned}
& \varepsilon\left(q+T \tilde{r}_{k}^{ \pm}+O\left(T^{2}\right)\right)=T \tilde{r}_{k}^{ \pm} \varepsilon_{0}^{\prime}+O\left(T^{2}\right)=2 \pi i T(k+1 / 2), \\
& \varepsilon\left(-q+T \tilde{r}_{k}^{ \pm}+O\left(T^{2}\right)\right)=-T \tilde{r}_{k}^{ \pm} \varepsilon_{0}^{\prime}+O\left(T^{2}\right)=2 \pi i T(k+1 / 2) .
\end{aligned}
$$

Thus, in the linear approximation in $T$, we obtain two series of roots

$$
\begin{cases}r_{k}^{+}= \pm q+\frac{2 \pi i T}{\varepsilon_{0}^{\prime}}(k+1 / 2)+O\left(T^{2}\right), & k \geq 0, \\ r_{k}^{-}= \pm q+\frac{2 \pi i T}{\varepsilon_{0}^{\prime}}(k+1 / 2)+O\left(T^{2}\right), & k<0 .\end{cases}
$$

We will refer to the roots collapsing to $+q$ as the right series and the roots collapsing to $-q$ as the left series. 


\subsection{Low-temperature limit of the $u(\lambda)$ integral equation}

From now on, we focus on a fixed contour $\hat{\mathcal{C}}_{i}$ and consider the associated contribution to the asymptotic behavior of the generating function. Therefore, below, we will omit the subscript $i$ in the notations of the contour $\hat{\mathcal{C}}_{i}$ and of the functions $u_{i}(\lambda), z_{i}(\lambda)$, etc associated with it.

Let $\hat{\mathcal{C}}$ be the contour bypassing $n$ points $\hat{s}^{+}$in the upper half-plane from above and $n$ points $\hat{s}^{-}$in the lower half-plane from below, where $n$ is an arbitrary, but fixed non-negative integer. These points $\hat{s}^{ \pm}$are roots of the equation $1+e^{-u\left(\hat{s}^{ \pm}\right) / T}=0$. It is important for our purpose to fix the limits of these roots at $T=0$.

The $T \rightarrow 0$ limit of equation (2.4) coincides with the one of the Yang-Yang equation, hence $\left.u(\lambda)\right|_{T=0}=\varepsilon_{0}(\lambda)$. Therefore it is reasonable to expect that, similarly to the points $r_{k}^{ \pm}$, the roots $\hat{s}_{k}^{ \pm}$collapse to $q$ or to $-q$ in the $T \rightarrow 0$ limit. Thus, in the low-temperature limit these roots should form two series. There will be

- $n_{p}^{+}$roots $\hat{s}^{+}$and $n_{h}^{+}$roots $\hat{s}^{-}$belonging to the right series;

- $n_{p}^{-}$roots $\hat{s}^{+}$and $n_{h}^{-}$roots $\hat{s}^{-}$belonging to the left series.

Obviously, there exists an integer $\ell,-n \leq \ell \leq n$, such that the numbers $n_{p}^{ \pm}$and $n_{h}^{ \pm}$are related by

$$
n_{p}^{+}+n_{p}^{-}=n_{h}^{+}+n_{h}^{-}=n, \quad n_{p}^{+}-n_{h}^{+}=n_{h}^{-}-n_{p}^{-}=\ell .
$$

Therefore, for $T$ small enough, one deals with the following structure for the distribution of roots $\hat{s}^{ \pm}$:

$$
\begin{array}{ll}
\left\{\hat{s}^{+}\right\}=\left\{q+i T \hat{\eta}_{k}^{+}\right\}_{n_{p}^{+}} \cup\left\{-q+i T \hat{\eta}_{k}^{-}\right\}_{n_{p}^{-}}, & \Re\left(\hat{\eta}_{k}^{ \pm}\right)>0, \\
\left\{\hat{s}^{-}\right\}=\left\{q-i T \hat{\xi}_{k}^{+}\right\}_{n_{h}^{+}} \cup\left\{-q-i T \hat{\xi}_{k}^{-}\right\}_{n_{h}^{-}}, & \Re\left(\hat{\xi}_{k}^{ \pm}\right)>0 .
\end{array}
$$

The parameters $\hat{\eta}_{k}^{ \pm}$and $\hat{\xi}_{k}^{ \pm}$admit the Taylor expansions $\hat{\eta}_{k}^{ \pm}=\eta_{k}^{ \pm}+\mathrm{O}(T)$ and $\hat{\xi}_{k}^{ \pm}=\xi_{k}^{ \pm}+\mathrm{O}(T)$. They appear in the non-linear integral equation (2.4) defining $u(\lambda)$ and should be computed by solving the conditions

$$
\exp \left(-\frac{u\left( \pm q+i T \hat{\eta}_{k}^{ \pm}\right)}{T}\right)=\exp \left(-\frac{u\left( \pm q-i T \hat{\xi}_{k}^{ \pm}\right)}{T}\right)=-1
$$

Substituting the parameterizations (3.13), (3.14) into the equation (2.4) and expanding up to the second order in $T$ we are led to

$$
u(\lambda)=\lambda^{2}-h-\frac{T}{2 \pi} \int_{\mathbb{R}} K(\lambda-\mu) \log \left(1+e^{-\frac{u(\mu)}{T}}\right) d \mu+T G_{1}(\lambda)+T^{2} G_{2}(\lambda)+O\left(T^{3}\right) .
$$

Here

$$
G_{1}(\lambda)=-2 \pi i \alpha-i \ell \int_{-q}^{q} K(\lambda-\mu) d \mu
$$


and

$$
G_{2}(\lambda)=K(\lambda-q)\left(\sum_{j=1}^{n_{p}^{+}} \eta_{j}^{+}+\sum_{j=1}^{n_{h}^{+}} \xi_{j}^{+}\right)+K(\lambda+q)\left(\sum_{j=1}^{n_{p}^{-}} \eta_{j}^{-}+\sum_{j=1}^{n_{h}^{-}} \xi_{j}^{-}\right) .
$$

It is natural to expect that the solution to (3.16) has a form similar to (3.7)

$$
u(\lambda)=\sum_{k=0}^{2} T^{k} u_{k}(\lambda)+O\left(T^{3}\right),
$$

where, as we have already argued, $u_{0}(\lambda)=\varepsilon_{0}(\lambda)$. Substituting (3.19) into (3.16) and using (A.10), we obtain linear integral equations satisfied by the unknown functions $u_{1}(\lambda)$ and $u_{2}(\lambda)$ :

$$
\begin{gathered}
u_{1}(\lambda)-\frac{1}{2 \pi} \int_{-q}^{q} K(\lambda-\mu) u_{1}(\mu) d \mu=G_{1}(\lambda), \\
u_{2}(\lambda)-\frac{1}{2 \pi} \int_{-q}^{q} K(\lambda-\mu) u_{2}(\mu) d \mu=G_{2}(\lambda)-\frac{\pi}{12 \varepsilon_{0}^{\prime}}(K(\lambda-q)+K(\lambda+q)) \\
-\frac{K(\lambda-q) u_{1}^{2}(q)}{4 \pi \varepsilon_{0}^{\prime}}-\frac{K(\lambda+q) u_{1}^{2}(-q)}{4 \pi \varepsilon_{0}^{\prime}} .
\end{gathered}
$$

It is then easy to see that the function $u_{1}(\lambda)-2 \pi i \ell$ satisfies (up to a multiplicative factor) the equation (3.3) for the total density at $T=0$. As we have already mentioned, on has that $2 \pi \rho_{t}(\lambda)=Z(\lambda)$ in the case of the QNLS model, with $Z(\lambda)$ being the dressed charge. Hence,

$$
u_{1}(\lambda)=u_{1}(-\lambda)=-2 \pi i \alpha_{\ell} Z(\lambda)+2 \pi i \ell, \quad \alpha_{\ell}=\alpha+\ell .
$$

The solution to equation (3.21) can be expressed in terms of the resolvent $R(\lambda, \mu)$ (3.5)

$$
\begin{aligned}
u_{2}(\lambda)=R(\lambda, q)\left[2 \pi \sum_{j=1}^{n_{p}^{+}} \eta_{j}^{+}\right. & \left.+2 \pi \sum_{j=1}^{n_{h}^{+}} \xi_{j}^{+}-\frac{1}{2 \varepsilon_{0}^{\prime}}\left(\frac{\pi^{2}}{3}+u_{1}^{2}(q)\right)\right] \\
+ & R(\lambda,-q)\left[2 \pi \sum_{j=1}^{n_{p}^{-}} \eta_{j}^{-}+2 \pi \sum_{j=1}^{n_{h}^{-}} \xi_{j}^{-}-\frac{1}{2 \varepsilon_{0}^{\prime}}\left(\frac{\pi^{2}}{3}+u_{1}^{2}(q)\right)\right] .
\end{aligned}
$$

It remains to fix the leading Taylor coefficients $\eta_{k}^{ \pm}$and $\xi_{k}^{ \pm}$. These can be parameterized by sets of integers, exactly as it was the case for the roots $r_{k}^{ \pm}$(3.11). More precisely, one has

$$
\begin{aligned}
& u\left( \pm q+i T \hat{\eta}_{k}^{ \pm}\right)= \pm 2 \pi i T\left(p_{k}^{ \pm}-\frac{1}{2}\right), \\
& u\left( \pm q-i T \hat{\xi}_{k}^{ \pm}\right)=\mp 2 \pi i T\left(h_{k}^{ \pm}-\frac{1}{2}\right),
\end{aligned}
$$


where $p_{k}^{ \pm}$and $h_{k}^{ \pm}$are integers. As a consequence, in the linear order in $T$, we obtain

$$
\begin{aligned}
& \varepsilon_{0}^{\prime} \eta_{k}^{ \pm}=2 \pi\left(p_{k}^{ \pm}-\frac{1}{2}\right) \pm i u_{1}(q), \\
& \varepsilon_{0}^{\prime} \xi_{k}^{ \pm}=2 \pi\left(h_{k}^{ \pm}-\frac{1}{2}\right) \mp i u_{1}(q),
\end{aligned}
$$

where $u_{1}(\lambda)$ is given by (3.22).

Remark. Let $u_{1} \equiv u_{1}( \pm q)=2 \pi i\left(\ell-\alpha_{\ell} \mathcal{Z}\right)$. From now on we assume that $u_{1}$ satisfies the constraint

$$
-\pi<\Im\left(u_{1}\right)<\pi
$$

Note that the generating function (1.3) is periodic over $\alpha$ [23]: $\left\langle e^{2 \pi i \alpha \mathcal{Q}_{x}}\right\rangle_{T}=\left\langle e^{2 \pi i(\alpha+1) \mathcal{Q}_{x}}\right\rangle_{T}$. Due to this property the condition above always can be satisfied by appropriate choice of the parameter $\alpha$. Therefore the constraint (3.26) does not imply any additional restrictions for the parameters of the model.

We stress that the condition (3.26) is purely technical. It allows us to simplify some intermediate calculations. In particular, it follows from (3.26) that all the integers $p_{k}^{ \pm}$and $h_{k}^{ \pm}$in (3.25) are positive. However, one can proceed further without use of the inequality (3.26).

Thus, in this way, we have found the solution $u(\lambda)$ to the equation (2.4) up to $O\left(T^{2}\right)$ terms and the roots $\hat{s}_{k}^{ \pm}$up to $O(T)$ terms. There is no fundamental obstacle to finding higher order corrections to $u(\lambda)$ and $\hat{s}_{k}^{ \pm}$. However, for our purposes, the results obtained here are already sufficient.

\section{Correlation lengths}

In this section we compute the rate $p[u]$ of the correlation function exponential decay. In the case of the QNLS model, the conformal dimensions giving rise to the critical exponents in the asymptotic expansion (1.5) were calculated in [24, 25]. We now obtain these results by taking the $T \rightarrow 0$ limit of equation (2.10).

We have already shown in the work [1] how the trivial constant term in (1.5) can be deduced from our approach to the asymptotics at finite temperature. More precisely, this constant stems from the contribution of the contour $\hat{\mathcal{C}}=\mathbb{R}$, in other words the case where the sets of the roots $\left\{\hat{s}^{ \pm}\right\}$are empty $(n=0)$. Therefore, in the following, we will only consider the case of non-empty sets $\left\{\hat{s}^{ \pm}\right\}$(although the results of our analysis remain valid for $n=0$ as well).

By moving the contour $\hat{\mathcal{C}}$ to the real axis, equation (2.10) boils down to

$$
p[u]=i \int_{\mathbb{R}} z(\mu) d \mu-i \sum_{k=1}^{n}\left(\hat{s}_{k}^{+}-\hat{s}_{k}^{-}\right) .
$$

The integral over $\mathbb{R}$ can be estimated to the leading order in $T$ with the help of (A.8), (A.10). In its turn, the finite sum is estimated directly by inserting the Taylor expansions of the roots 
$\hat{s}_{k}^{ \pm}$. Ultimately, one gets that, to the linear order in $T$,

$$
p[u]=-2 i \alpha_{\ell} k_{F}-\frac{T \mathcal{Z} u_{1}^{2}}{2 \pi \varepsilon_{0}^{\prime}}+T \mathcal{Z}\left(\sum_{j=1}^{n_{p}^{+}} \eta_{j}^{+}+\sum_{j=1}^{n_{p}^{-}} \eta_{j}^{-}+\sum_{j=1}^{n_{h}^{+}} \xi_{j}^{+}+\sum_{j=1}^{n_{h}^{-}} \xi_{j}^{-}\right)+O\left(T^{2}\right) .
$$

where we have used (3.4) and (3.6). We recall also that $u_{1}=u_{1}(q)=2 \pi i\left(\ell-\alpha_{\ell} \mathcal{Z}\right)$. Finally, it remains to use that $\eta_{k}^{ \pm}, \xi_{k}^{ \pm}$are given by (3.25). This leads to

$$
p[u]=-2 i \alpha_{\ell} k_{F}+\frac{2 \pi T}{v_{0}}\left[\left(\alpha_{\ell} \mathcal{Z}\right)^{2}-\ell^{2}-n+\sum_{j=1}^{n_{p}^{+}} p_{j}^{+}+\sum_{j=1}^{n_{p}^{-}} p_{j}^{-}+\sum_{j=1}^{n_{h}^{+}} h_{j}^{+}+\sum_{j=1}^{n_{h}^{-}} h_{j}^{-}\right]+O\left(T^{2}\right) .
$$

\section{Constant amplitude}

In this section, we compute the low-temperature limit of the constant coefficients $B_{d}[u]$ (2.11) and $B_{s}[u]$ (2.14). We prove that in this limit, when properly normalized in the temperature, $B[u]$ goes to the amplitude of a critical form factor. The latter form factors correspond to expectation values of local operators taken between the ground state and excited states where all rapidities of the particles and holes are located on the Fermi boundary. We first recall several definitions and results concerning the form factors in the QNLS model. The reader can find a more detailed exposition in [17]

The form factors of the QNLS model can be parameterized by the rapidities of particles and holes [26, 27, 28, 29]. If, in the thermodynamic limit $(L \rightarrow \infty)$ all the rapidities are located on the Fermi boundaries $\pm q$, then the corresponding form factor is called critical form factor [17]. Hereby the distribution of the rapidities between $+q$ and $-q$ is important.

Consider a critical form factor parameterized by the rapidities of $n$ particles and $n$ holes. Assume that, in the thermodynamic limit, there is $n_{p}^{+}$(resp. $n_{h}^{+}$) rapidities of the particles (resp. holes) going to $+q$ and $n_{p}^{-}$(resp. $n_{h}^{-}$) rapidities of the particles (resp. holes) going to $-q$. We say that a given form factor belongs to the $\mathbf{P}_{\ell}$ class, if the numbers $n_{p, h}^{ \pm}$satisfy the conditions gathered in (3.12), with $\ell$ being some fixed integer.

The critical form factors can be presented as a product of a smooth and a discrete part (see [17]). The smooth part has a well defined thermodynamic limit $L \rightarrow \infty$. The discrete part, strictly speaking, has no thermodynamic limit. First of all, it scales to zero as some negative power of $L$, when $L \rightarrow \infty$. Second, it not only depends on the rapidities of the particles and holes (which are equal to $\pm q$ ), but also on the quantum numbers associated with the excited state.

In the following, we show that the factor $B_{d}[u]$ in (2.11) exactly reproduces the discrete part of the critical form factor of the $\mathbf{P}_{\ell}$ class, provided the distribution (3.12) is fixed. Hereby the

\footnotetext{
${ }^{1}$ Formally the work [17] deals with form factors of the $X X Z$ spin chain, however the results obtained there can be easily reduced to the case of the QNLS model.
} 
role of large $L$ is played by the inverse temperature: $v_{0} / i T \leftrightarrow L$. The integers $p_{j}^{ \pm}$and $h_{j}^{ \pm}$(see (3.24)) play the role of the quantum numbers describing particles and holes.

The coefficient $B_{s}[u](2.14)$ gives the smooth part of the critical form factor. We first focus on the analysis related with $B_{s}[u]$ as the computation of its $T \rightarrow 0$ limit is simpler then for $B_{d}[u]$.

\subsection{Smooth part}

The coefficient $B_{s}[u]$ can be seen as mostly depending on integrals of the following type:

$$
I_{f}=\int_{\hat{\mathcal{C}}} f^{\prime}(\lambda) z(\lambda) d \lambda,
$$

where $z(\lambda)$ is given by (2.9) and $f(\lambda)$ is holomorphic in some domain containing $\hat{\mathcal{C}}$ and $\mathbb{R}$. Then moving $\hat{\mathcal{C}}$ to $\mathbb{R}$ we obtain

$$
I_{f} \rightarrow \int_{\mathbb{R}} f^{\prime}(\lambda) z(\lambda) d \lambda-\ell(f(q)-f(-q)), \quad T \rightarrow 0,
$$

since all roots $\left\{\hat{s}^{ \pm}\right\}$go to $\pm q$ at $T \rightarrow 0$. Using that, at $T=0 z(\lambda)=0$ for $|\lambda|>q$ and $z(\lambda)=u_{1}(\lambda) / 2 \pi i$ for $|\lambda|<q$, we find

$$
\lim _{T \rightarrow 0} \int_{\mathbb{R}} f^{\prime}(\lambda) z(\lambda) d \lambda=\frac{1}{2 \pi i} \int_{-q}^{q} f^{\prime}(\lambda) u_{1}(\lambda) d \lambda,
$$

and hence, due to (3.22)

$$
\lim _{T \rightarrow 0} \int_{\hat{\mathcal{C}}} f^{\prime}(\lambda) z(\lambda) d \lambda=-\alpha_{\ell} \int_{-q}^{q} f^{\prime}(\lambda) Z(\lambda) d \lambda .
$$

Using this prescription we obtain for the limit of the Cauchy transforms

$$
\lim _{T \rightarrow 0} L_{\hat{\mathcal{C}}}[z](w+i \gamma c)=-\alpha_{\ell} L_{[-q, q]}[Z](w+i \gamma c), \quad \gamma=0, \pm 1 .
$$

Similarly

$$
\lim _{T \rightarrow 0} C_{0}[z]=\alpha_{\ell}^{2} \int_{-q}^{q} \frac{Z(\lambda) Z(\mu)}{(\lambda-\mu-i c)^{2}} d \lambda d \mu .
$$

Another type of integrals arises in the Fredholm determinant $\operatorname{det}_{\hat{\mathcal{C}}}\left[I-\frac{1}{2 \pi} K^{(u)}\right]$. Recall that this operator acts on the contour $\hat{\mathcal{C}}$ as

$$
\left[I-\frac{1}{2 \pi} K^{(u)}\right] f(\lambda)=f(\lambda)-\frac{1}{2 \pi} \int_{\hat{\mathcal{C}}} K^{(u)}(\lambda, \mu) f(\mu) d \mu,
$$


where $K^{(u)}(\lambda, \mu)$ is given by (2.15). If $f(\lambda)$ is holomorphic in a domain containing $\hat{\mathcal{C}}$ and $\mathbb{R}$, then one can easily see that

$$
\int_{\hat{\mathcal{C}}} K^{(u)}(\lambda, \mu) f(\mu) d \mu=\int_{\mathbb{R}} K^{(u)}(\lambda, \mu) f(\mu) d \mu+O(T), \quad T \rightarrow 0 .
$$

Since $u(\lambda)=\varepsilon_{0}(\lambda)$ at $T=0$ we conclude that, in the $T \rightarrow 0$ limit, the action of the operator $I-\frac{1}{2 \pi} K^{(u)}$ coincides with the one of $I-\frac{1}{2 \pi} K^{(\varepsilon)}$. The action of this last operator clearly reduces to the interval $[-q, q]$ when $T=0$. Thus,

$$
\lim _{T \rightarrow 0} \operatorname{det}_{\mathbb{R}}\left[I-\frac{1}{2 \pi} K^{(\varepsilon)}\right]=\lim _{T \rightarrow 0} \operatorname{det}_{\hat{\mathcal{C}}}\left[I-\frac{1}{2 \pi} K^{(u)}\right]=\operatorname{det}_{[-q, q]}\left[I-\frac{1}{2 \pi} K\right] .
$$

Substituting all these results into (2.14) we immediately reproduce the smooth part of the critical form factor obtained in [17]. We give these rather cumbersome expressions in appendix C]

\subsection{Discrete part}

The $T \rightarrow 0$ limit of the factor $B_{d}[u]$ (2.11) is more involved. In order to compute it, we first deform the contour $\hat{\mathcal{C}}$ to the real axis. This provides an alternative expression for $B_{d}[u]$, that was originally obtained in 30 ]

$$
\begin{aligned}
B_{d}[u]=\exp ( & \left.\int_{\mathbb{R}} \frac{z(\lambda) z(\mu)}{\left(\lambda-\mu_{+}\right)^{2}} d \lambda d \mu\right) \cdot\left(\operatorname{det} \frac{1}{\hat{s}_{j}^{+}-\hat{s}_{k}^{-}}\right)^{2} \\
& \times \prod_{j=1}^{n} e^{2 L_{\mathbb{R}}[z]\left(\hat{s}_{j}^{-}\right)-2 L_{\mathbb{R}}[z]\left(\hat{s}_{j}^{+}\right)}\left(\left.\partial_{\lambda} e^{-2 \pi i z(\lambda)}\right|_{\lambda=\hat{s}_{j}^{-}}\right)^{-1}\left(\left.\partial_{\lambda} e^{-2 \pi i z(\lambda)}\right|_{\lambda=\hat{s}_{j}^{+}}\right)^{-1} .
\end{aligned}
$$

Consider the behavior of the Cauchy determinant in (5.10) at $T \rightarrow 0$. We have

$$
\left(\operatorname{det}_{n} \frac{1}{\hat{s}_{j}^{+}-\hat{s}_{k}^{-}}\right)^{2}=\frac{\prod_{j>k}^{n}\left(\hat{s}_{j}^{+}-\hat{s}_{k}^{+}\right)^{2}\left(\hat{s}_{j}^{-}-\hat{s}_{k}^{-}\right)^{2}}{\prod_{j, k=1}^{n}\left(\hat{s}_{j}^{+}-\hat{s}_{k}^{-}\right)^{2}} .
$$

Now we should substitute here (3.13), (3.14) and (3.25). Hereby at $T \rightarrow 0$ we can set $\left(\hat{s}_{j}^{ \pm}-\hat{s}_{k}^{ \pm}\right)^{2}=$ $\left(\hat{s}_{j}^{ \pm}-\hat{s}_{k}^{\mp}\right)^{2}=4 q^{2}$, if the roots belong to the different series. Then we obtain

$$
\begin{aligned}
& \lim _{T \rightarrow 0}\left(T^{n-\ell^{2}} \operatorname{det}_{n} \frac{1}{\hat{s}_{j}^{+}-\hat{s}_{k}^{-}}\right)^{2}=(-1)^{n+\ell}\left(\frac{q \varepsilon_{0}^{\prime}}{\pi}\right)^{-2 \ell^{2}}\left(\frac{\varepsilon_{0}^{\prime}}{2 \pi}\right)^{2 n} \\
& \times \frac{\prod_{j>k}^{n_{p}^{+}}\left(p_{j}^{+}-p_{k}^{+}\right)^{2} \prod_{j>k}^{n_{h}^{+}}\left(h_{j}^{+}-h_{k}^{+}\right)^{2} \prod_{j>k}^{n_{p}^{-}}\left(p_{j}^{-}-p_{k}^{-}\right)^{2} \prod_{j>k}^{n_{h}^{-}}\left(h_{j}^{-}-h_{k}^{-}\right)^{2}}{\prod_{j=1}^{n_{p}^{+}} \prod_{k=1}^{n_{h}^{+}}\left(p_{j}^{+}+h_{k}^{+}-1\right)^{2}} . \\
& \prod_{j=1}^{n_{p}^{-}} \prod_{k=1}^{n_{h}^{-}}\left(p_{j}^{-}+h_{k}^{-}-1\right)^{2}
\end{aligned}
$$


Consider now the low-temperature behavior of the derivatives $\partial_{\lambda} e^{-2 \pi i z(\lambda)}$ at $\lambda=\hat{s}_{j}^{ \pm}$. We have

$$
e^{-2 \pi i z(\lambda)}=\frac{1+e^{-\frac{u(\lambda)}{T}}}{1+e^{-\frac{\varepsilon(\lambda)}{T}}}
$$

Since $1+e^{-\frac{u\left(\hat{s}_{j}^{ \pm}\right)}{T}}=0$ we obtain

$$
\left.T \partial_{\lambda} e^{-2 \pi i z(\lambda)}\right|_{\lambda=\hat{s}_{j}^{ \pm}}=\frac{-u^{\prime}\left(\hat{s}_{j}^{ \pm}\right)}{1+e^{-\frac{\varepsilon\left(\hat{s}_{j}^{ \pm}\right)}{T}}}=\frac{-u^{\prime}\left(\hat{s}_{j}^{ \pm}\right)}{1-e^{-\frac{\varepsilon\left(\hat{s}_{j}^{ \pm}\right)-u\left(\hat{s}_{j}^{ \pm}\right)}{T}}} .
$$

Substituting here (3.19), (3.22) we arrive at

$$
\left.\lim _{T \rightarrow 0} T \partial_{\lambda} e^{-2 \pi i z(\lambda)}\right|_{\lambda=\hat{s}_{j}^{ \pm}}=\left\{\begin{array}{cl}
-\varepsilon_{0}^{\prime}\left(1-e^{-2 \pi i \alpha_{\ell} \mathcal{Z}}\right)^{-1}, & \text { if } \hat{s}_{j}^{ \pm} \text {belongs to the right series } \\
\varepsilon_{0}^{\prime}\left(1-e^{-2 \pi i \alpha_{\ell} \mathcal{Z}}\right)^{-1}, & \text { if } \hat{s}_{j}^{ \pm} \text {belongs to the left series. }
\end{array}\right.
$$

The estimate of the $T \rightarrow 0$ behavior of the Cauchy transforms $L_{\mathbb{R}}[z]\left(\hat{s}_{j}^{ \pm}\right)$is a more complicated problem. It is easy to see that one cannot use (5.5) in this case. Indeed, on the one hand the Cauchy transform $L_{[-q, q]}[z](\omega)$ on the interval $[-q, q]$ has a logarithmic singularity at $\omega= \pm q$. On the other hand, we have seen that all $\hat{s}_{j}^{ \pm}$go to $\pm q$ at $T \rightarrow 0$, therefore $L_{\mathbb{R}}[z]\left(\hat{s}_{j}^{ \pm}\right)$ should diverge as a multiple of $\log T$ in the low-temperature limit. In fact, the equation (5.5) only allows one to access to this divergent part; however it does not give an access to the finite part of the $T \rightarrow 0$ behavior of the Cauchy transform.

Similar problem occurs at studying the $T \rightarrow 0$ behavior of the double integral

$$
A=\int_{\mathbb{R}} \frac{z(\lambda) z(\mu)}{\left(\lambda-\mu_{+}\right)^{2}} d \lambda d \mu
$$

The above double integral can be reduced to a single one

$$
A=\int_{\mathbb{R}} \frac{z(\lambda) z(\mu)}{\left(\lambda-\mu_{+}\right)^{2}} d \lambda d \mu=\int_{\mathbb{R}}\left(\partial_{\mu} L_{\mathbb{R}}[z]\left(\mu_{+}\right)\right) \cdot z(\mu) d \mu .
$$

Thus, in order to estimate this integral at $T \rightarrow 0$ one should know the behavior of $L_{\mathbb{R}}[z](\mu)$ on the whole real axis including the points $\pm q$. This question is studied in details in appendix B Here we restrict ourselves to present the results of these computations.

First of all, we list the leading $T \rightarrow 0$ behavior of the exponents of the Cauchy transforms $L_{\mathbb{R}}[z]\left(\hat{s}_{j}^{ \pm}\right)$. There are four cases to distinguish:

- $\hat{s}_{k}^{+}$belongs to the right or to the left series,

- $\hat{s}_{k}^{-}$belongs to the right or to the left series. 
Using the parametrization (3.13), (3.14) and (3.25) we have

$$
\begin{aligned}
& \lim _{T \rightarrow 0}\left\{e^{L_{\mathbb{R}}[z]\left( \pm q+i T \hat{\eta}_{k}^{ \pm}\right)}\left(\frac{q \varepsilon_{0}^{\prime}}{\pi T}\right)^{ \pm \frac{u_{1}}{2 \pi i}}\right\}=\exp \left\{-\alpha_{\ell} \int_{-q}^{q} \frac{Z(\mu)-\mathcal{Z}}{\mu \mp q} d \mu-\frac{u_{1}}{4}\right\} \frac{\Gamma\left(p_{k}^{ \pm}\right)}{\Gamma\left(p_{k}^{ \pm} \mp \frac{u_{1}}{2 \pi i}\right)}, \\
& \lim _{T \rightarrow 0}\left\{e^{L_{\mathbb{R}}[z]\left( \pm q-i T \hat{\xi}_{k}^{ \pm}\right)}\left(\frac{q \varepsilon_{0}^{\prime}}{\pi T}\right)^{ \pm \frac{u_{1}}{2 \pi i}}\right\}=\exp \left\{-\alpha_{\ell} \int_{-q}^{q} \frac{Z(\mu)-\mathcal{Z}}{\mu \mp q} d \mu+\frac{u_{1}}{4}\right\} \frac{\Gamma\left(h_{k}^{ \pm} \pm \frac{u_{1}}{2 \pi i}\right)}{\Gamma\left(h_{k}^{ \pm}\right)} .
\end{aligned}
$$

Thus, for a given partition (3.13), (3.14) of roots $\hat{s}_{j}^{ \pm}$into the right and left series, we obtain

$$
\begin{aligned}
& \lim _{T \rightarrow 0} \prod_{j=1}^{n} e^{2 L_{\mathbb{R}}[z]\left(\hat{s}_{j}^{-}\right)-2 L_{\mathbb{R}}[z]\left(\hat{s}_{j}^{+}\right)}\left(\frac{q \varepsilon_{0}^{\prime}}{\pi T}\right)^{-\frac{2 \ell u_{1}}{\pi i}}=\exp \left\{4 \ell \alpha_{\ell} \int_{-q}^{q} \frac{Z(\mu)-\mathcal{Z}}{\mu-q} d \mu+2 i \pi n \alpha_{\ell} \mathcal{Z}\right\} \\
& \times \Gamma^{2}\left(\begin{array}{cccc}
\left\{p_{k}^{+}-\frac{u_{1}}{2 \pi i}\right\}, & \left\{h_{k}^{+}+\frac{u_{1}}{2 \pi i}\right\}, & \left\{p_{k}^{-}+\frac{u_{1}}{2 \pi i}\right\}, & \left\{h_{k}^{-}-\frac{u_{1}}{2 \pi i}\right\} \\
\left\{p_{k}^{+}\right\}, & \left\{h_{k}^{+}\right\}, & \left\{p_{k}^{-}\right\}, & \left\{h_{k}^{-}\right\}
\end{array}\right),
\end{aligned}
$$

where we use the standard hypergeometric type notation for ratios of $\Gamma$-functions:

$$
\Gamma\left(\begin{array}{c}
a_{1}, \ldots, a_{p} \\
b_{1}, \ldots, b_{q}
\end{array}\right)=\prod_{k=1}^{p} \Gamma\left(a_{k}\right) \cdot \prod_{k=1}^{q} \Gamma\left(b_{k}\right)^{-1} .
$$

In its turn, the exponent of the double integral (5.16) exhibits the following leading $T \rightarrow 0$ behavior

$$
\lim _{T \rightarrow 0} \exp \left(\int_{\mathbb{R}} \frac{z(\lambda) z(\mu)}{\left(\lambda-\mu_{+}\right)^{2}} d \lambda d \mu\right)\left(\frac{q \varepsilon_{0}^{\prime}}{\pi T}\right)^{-\frac{u_{1}^{2}}{2 \pi^{2}}}=e^{C_{1}\left[\frac{u_{1}(\lambda)}{2 \pi i}\right]} G^{2}\left(1, \frac{u_{1}}{2 \pi i}\right) .
$$

The functional $C_{1}[F]$ appearing above reads

$$
C_{1}[F(\lambda)]=\frac{1}{2} \int_{-q}^{q} \frac{F^{\prime}(\lambda) F(\mu)-F(\lambda) F^{\prime}(\mu)}{\lambda-\mu} d \lambda d \mu+2 F(q) \int_{-q}^{q} \frac{F(\lambda)-F(q)}{\lambda-q},
$$

and $G(1, x)=G(1+x) G(1-x)$, where $G(x)$ is the Barnes function.

Now we substitute $u_{1} / 2 \pi i=\ell-\alpha_{\ell} \mathcal{Z}$. Combining (5.12), (5.15), (5.20), and (5.22) and using

$$
C_{1}\left[\alpha_{\ell} Z(\lambda)-\ell\right]=C_{1}\left[\alpha_{\ell} Z(\lambda)\right]-4 \ell \alpha_{\ell} \int_{-q}^{q} \frac{Z(\mu)-\mathcal{Z}}{\mu-q} d \mu
$$

we obtain

$$
\begin{aligned}
B_{d}^{(0)}[u]=\lim _{T \rightarrow 0} B_{d}[u]\left|\frac{q \varepsilon_{0}^{\prime}}{i \pi T}\right|^{2 \alpha_{\ell}^{2} \mathcal{Z}^{2}}=e^{C_{1}\left[\alpha_{\ell} Z(\lambda)\right]}\left(\frac{\sin \pi \alpha_{\ell} \mathcal{Z}}{\pi}\right)^{2 n} G^{2}\left(1, \alpha_{\ell} \mathcal{Z}-\ell\right) \\
\\
\times R_{n_{p}^{+}, n_{h}^{+}}\left(\left\{p^{+}\right\},\left\{h^{+}\right\} \mid \alpha_{\ell} \mathcal{Z}-\ell\right) R_{n_{p}^{-}, n_{h}^{-}}\left(\left\{p^{-}\right\},\left\{h^{-}\right\} \mid \ell-\alpha_{\ell} \mathcal{Z}\right)
\end{aligned}
$$


where

$$
R_{n, m}(\{p\},\{h\} \mid \nu)=\frac{\prod_{j>k}^{n}\left(p_{j}-p_{k}\right)^{2} \prod_{j>k}^{m}\left(h_{j}-h_{k}\right)^{2}}{\prod_{j=1}^{n} \prod_{k=1}^{m}\left(p_{j}+h_{k}-1\right)^{2}} \Gamma^{2}\left(\begin{array}{c}
\left\{p_{k}+\nu\right\}, \\
\left\{p_{k}\right\},\left\{h_{k}-\nu\right\}
\end{array}\right) .
$$

We have thus reproduced the discrete part of form factors given in [17] up to the replacement $v_{0} / T \mapsto i L$.

\section{$6 \quad$ Final result}

We have calculated the low-temperature limits of the exponential decay and constant coefficient in the long-distance asymptotic expansion of the generating function $\left\langle e^{2 i \pi \alpha \mathcal{Q}_{x}}\right\rangle_{T}$ for given distribution of the roots $\hat{s}_{j}^{ \pm}$. In this section we sum up all the obtained contributions for $\ell$ fixed.

Let us first summarize the results obtained in the previous sections. The asymptotic expansion of the generating function $\left\langle e^{2 i \pi \alpha \mathcal{Q}_{x}}\right\rangle_{T}$ at low temperature has the form

$$
\left\langle e^{2 i \pi \alpha \mathcal{Q}_{x}}\right\rangle_{T} \simeq \sum_{\ell \in \mathbb{Z}} Q_{\ell} e^{2 i \alpha_{\ell} k_{F} x} \quad x \rightarrow \infty, \quad T \rightarrow 0, \quad x T \rightarrow \infty
$$

where the coefficients $Q_{\ell}$ are

$$
Q_{\ell}=B_{s}^{(0)}\left[\alpha_{\ell} Z\right]\left(\frac{\pi T}{q \varepsilon_{0}^{\prime}}\right)^{2 \alpha_{\ell}^{2} \mathcal{Z}^{2}} G^{2}\left(1, \alpha_{\ell} \mathcal{Z}-\ell\right) e^{C_{1}\left[\alpha_{\ell} Z(\lambda)\right]-\frac{2 \pi T x}{v_{0}}\left(\left(\alpha_{\ell} \mathcal{Z}\right)^{2}-\ell^{2}\right)} W_{+} W_{-} .
$$

Here the smooth part $B_{s}^{(0)}\left[\alpha_{\ell} Z\right]$ is given by ([C.1) , the functional $C_{1}$ by (‥23). To describe the factors $W_{ \pm}$we first define a function $W(\nu, r)$ depending on complex $\nu$ and integer $r$ as

$$
\begin{aligned}
& W(\nu, r)=\sum_{\substack{n, n^{\prime}=0 \\
n-n^{\prime}=r}}^{\infty} \sum_{\substack{1 \leq p_{1}<\cdots<p_{n}<\infty \\
1 \leq h_{1}<\cdots<h_{n^{\prime}}<\infty}} \prod_{j=1}^{n} e^{-\frac{2 \pi T x}{v_{0}}\left(p_{j}-1\right)} \prod_{k=1}^{n^{\prime}} e^{-\frac{2 \pi T x}{v_{0}} h_{k}} \\
& \times\left(\frac{\sin \pi \nu}{\pi}\right)^{2 n^{\prime}} R_{n, n^{\prime}}(\{p\},\{h\} \mid \nu),
\end{aligned}
$$

where $R_{n, n^{\prime}}(\{p\},\{h\} \mid \nu)$ is given by (15.26). Then

$$
W_{ \pm}=\left.W(\nu, r)\right|_{\substack{\nu= \pm\left(\alpha_{\ell} \mathcal{Z}-\ell\right), r= \pm \ell}},
$$

It is easy to see that the factors $W_{ \pm}$correspond to the sums over all the possible choices of integers $\left\{p_{a}^{ \pm}\right\}_{1}^{n_{p}^{ \pm}}$and $\left\{h_{a}^{ \pm}\right\}_{1}^{n_{h}^{ \pm}}$which parameterize the possible configurations of the roots $\hat{s}_{j}^{ \pm}$at $\ell$ fixed. Such sums are computed in [31] as,

$$
W(\nu, r)=\frac{G^{2}(1+r+\nu)}{G^{2}(1+\nu)} \frac{e^{-\frac{\pi T x}{v_{0}} r(r-1)}}{\left(1-e^{-\frac{2 \pi T x}{v_{0}}}\right)^{(\nu+r)^{2}}} .
$$


Setting here $\nu= \pm\left(\alpha_{\ell} \mathcal{Z}-\ell\right), r= \pm \ell$ and substituting (6.5) into (6.2) we obtain after simple algebra

$$
Q_{\ell}=\widetilde{A}_{\ell}\left(\frac{\pi T / v_{0}}{\sinh \frac{\pi T x}{v_{0}}}\right)^{2 \alpha_{\ell}^{2} \mathcal{Z}^{2}}, \quad \text { with } \quad \widetilde{A}_{\ell}=B_{s}^{(0)}\left[\alpha_{\ell} Z\right] \frac{G^{2}\left(1, \alpha_{\ell} \mathcal{Z}\right)}{(2 q \mathcal{Z})^{2 \alpha_{\ell}^{2} \mathcal{Z}^{2}}} e^{C_{1}\left[\alpha_{\ell} Z(\lambda)\right]}
$$

where we have used $\varepsilon_{0}^{\prime}=\mathcal{Z} v_{0}$. Thus we arrive at the following asymptotic expansion

$$
\left\langle e^{2 i \pi \alpha \mathcal{Q}_{x}}\right\rangle_{T} \simeq \sum_{\ell \in \mathbb{Z}} e^{2 i \alpha_{\ell} k_{F} x}\left(\frac{\pi T / v_{0}}{\sinh \frac{\pi T x}{v_{0}}}\right)^{2 \alpha_{\ell}^{2} \mathcal{Z}^{2}} \widetilde{A}_{\ell} .
$$

Note that the obtained result is obviously a periodic function of $\alpha$ as it was expected thanks to the fact that the coefficient $\widetilde{A}_{\ell}$ is also a function of $\alpha_{\ell}$ only, see appendix [C] It is also straightforward to see that the combination $\widetilde{A}_{\ell}\left(\pi T / v_{0}\right)^{2 \ell^{2} \mathcal{Z}^{2}}$ coincides with the amplitude of the critical form factor of the operator $e^{2 i \pi \alpha \mathcal{Q}_{x}}$ [17] corresponding to the umklapp-type excited state of the momentum $2 \alpha_{\ell} k_{F}$, where $v_{0} / T$ plays the role of the system size.

Finally, in order to obtain the long-distance asymptotic expansion of the density-density correlation function it is enough to apply the differential operator (1.4) to the equation (6.7). Hereby one should distinguish two cases: $\ell=0$ and $\ell \neq 0$. In the last case one has due to (C.1)

$$
\left.B_{s}^{(0)}\left[\alpha_{\ell} Z\right]\right|_{\alpha=0}=0,\left.\quad \partial_{\alpha} B_{s}^{(0)}\left[\alpha_{\ell} Z\right]\right|_{\alpha=0}=0
$$

Therefore the second $\alpha$-derivative should be applied to the coefficient $B_{s}^{(0)}\left[\alpha_{\ell} Z\right]$.

On the contrary $B_{s}^{(0)}\left[\alpha_{\ell} Z\right]=1$ at $\alpha=0$ and $\ell=0$, that is at $\alpha_{\ell}=0$, (see [1]). Therefore in that case, the second $\alpha$-derivative should be applied on the combination containing the dependence on $x$, namely on $\exp \left[2 i \alpha_{\ell} k_{F} x\right]\left(\sinh \left(\pi T x / v_{0}\right)\right)^{-2 \alpha_{\ell}^{2} \mathcal{Z}^{2}}$, as otherwise the second $x$-derivative vanishes. Thus, taking the second $\alpha$ and $x$ derivatives of (6.7) as it is explained above, and neglecting higher order corrections over $T$ as well as subdominant exponential decays in $x$, we arrive at (1.5) with

$$
A_{\ell}=\left.\frac{D^{2} \ell^{2}}{2} \frac{\partial^{2}}{\partial \alpha^{2}} \widetilde{A}_{\ell}\right|_{\alpha=0} .
$$

It is readily checked that in the $x \rightarrow \infty, x T \rightarrow 0$ limit equation (1.5) does reproduce the longdistance asymptotic expansion of the density-density correlation function of the one dimensional Bose gas at $T=0$, together with the correct values of the amplitudes [19].

\section{Acknowledgements}

We are very grateful to N. Kitanine and V. Terras for useful and numerous discussions. J. M. M. and N. S. are supported by CNRS. We acknowledge the support from the GDRI-471 of CNRS "French-Russian network in Theoretical and Mathematical Physics" and RFBR-CNRS-09-0193106L-a. J. M. M. is also supported by the ANR grant DIADEMS 10 BLAN 012004, N. S. by 
the Program of RAS Mathematical Methods of the Nonlinear Dynamics, RFBR-11-01-00440a, SS-8265.2010.1 and K. K. K. by the EU Marie-Curie Excellence Grant MEXT-CT-2006-042695. N. S. and K. K. K would like to thank the Theoretical Physics group of the Laboratory of Physics at ENS Lyon for hospitality, which makes this collaboration possible.

\section{A Estimates of integrals with regular functions}

In this appendix we estimate the class of integrals that appears in equations (2.1) and (2.4). We first focus on the integrals of the form:

$$
J[\varepsilon]=T \int_{\mathbb{R}} f(\lambda) \log \left(1+e^{-\frac{\varepsilon(\lambda)}{T}}\right) d \lambda .
$$

For our purpose, it is enough to consider the case when $f(\lambda)$ is bounded on the real axis and differentiable in vicinities of $\pm q$, although the result of the analysis remains valid at much less restrictive assumptions. Due to the properties of $\varepsilon(\lambda)(3.1)$ it is clear that

$$
\lim _{T \rightarrow 0} J[\varepsilon]=-\int_{-q}^{q} f(\lambda) \varepsilon(\lambda) d \lambda .
$$

In order to find power-law corrections to the equation (A.2), one should estimate the contributions coming from the vicinities of $\pm q$ more thoroughly. Let $\delta>0$ be such that $\delta \rightarrow 0$ as $T \rightarrow 0$, while $\delta / T \rightarrow \infty$ as $T \rightarrow 0$. We can split the integral $J$ into five parts $J=J_{-}+J_{-q}+J_{0}+J_{q}+J_{+}$. The integrals $J_{ \pm}$correspond to the domains $\lambda>q+\delta$ and $\lambda<-q-\delta$. They behave as $O\left(e^{-\varepsilon( \pm q \pm \delta) / T}\right)$ and hence produce $O\left(T^{\infty}\right)$ contributions. The integral $J_{0}$ runs along the domain $-q+\delta<\lambda<q-\delta$. By factoring out $e^{-\frac{\varepsilon(\lambda)}{T}}$ from the logarithm, we get that

$$
J_{0}[\varepsilon]=-\int_{-q+\delta}^{q-\delta} f(\lambda) \varepsilon(\lambda) d \lambda+O\left(T^{\infty}\right) .
$$

Finally the integrals $J_{ \pm q}$ correspond to the domains $\pm q-\delta<\lambda< \pm q+\delta$ and generate all power-law corrections in $\mathrm{T}$ to (A.2). We now derive the leading power-law correction to A.2 coming from the $\delta$-vicinity of $q$. For doing this, we can replace the functions entering the integral by the leading non-vanishing terms of their Taylor expansions. Namely, we replace $f(\lambda)$ by $f(q)$ and $\varepsilon(\lambda)$ by $(\lambda-q) \varepsilon_{0}^{\prime}+T \varepsilon_{1}(q)$. Recall that $\varepsilon_{0}(q)=0$ and we denote $\varepsilon_{0}^{\prime} \equiv \varepsilon_{0}^{\prime}(q)$. Thence,

$$
J_{q}[\varepsilon]=T f(q) \int_{-\delta}^{\delta} \log \left(1+e^{-\frac{\lambda \varepsilon_{0}^{\prime}}{T}-\varepsilon_{1}(q)}\right) d \lambda+\text { h.o.c. }
$$


where h.o.c. means the higher order corrections in $T$. After changing of variables $\lambda=\mu T / \varepsilon_{0}^{\prime}$ we obtain

$$
\begin{aligned}
J_{q}[\varepsilon]=\frac{T^{2} f(q)}{\varepsilon_{0}^{\prime}} \int_{-\delta \varepsilon_{0}^{\prime} / T}^{\delta \varepsilon_{0}^{\prime} / T}\left[\log \left(1+e^{-\mu-\varepsilon_{1}(q)}\right)+\left(\mu+\varepsilon_{1}(q)\right) \Theta\left(-\mu-\varepsilon_{1}(q)\right)\right] d \mu \\
\\
-\frac{T^{2} f(q)}{\varepsilon_{0}^{\prime}} \int_{-\delta \varepsilon_{0}^{\prime} / T}^{-\varepsilon_{1}(q)}\left(\mu+\varepsilon_{1}(q)\right) d \mu+\text { h.o.c. }
\end{aligned}
$$

where $\Theta(\lambda)$ is the Heaviside step-function. Using now that $\delta / T \rightarrow \infty$ we arrive at

$$
\begin{array}{r}
J_{q}[\varepsilon]=\frac{T^{2} f(q)}{2 \varepsilon_{0}^{\prime}}\left(\varepsilon_{1}(q)-\frac{\delta \varepsilon_{0}^{\prime}}{T}\right)^{2}+\frac{T^{2} f(q)}{\varepsilon_{0}^{\prime}} \int_{-\infty}^{\infty}\left[\log \left(1+e^{-\mu}\right)+\mu \Theta(-\mu)\right] d \mu \\
=\frac{T^{2} f(q)}{2 \varepsilon_{0}^{\prime}}\left(\varepsilon_{1}(q)-\frac{\delta \varepsilon_{0}^{\prime}}{T}\right)^{2}+\frac{\pi^{2} T^{2} f(q)}{6 \varepsilon_{0}^{\prime}}+\text { h.o.c. } .
\end{array}
$$

Similarly one has

$$
J_{-q}[\varepsilon]=\frac{T^{2} f(-q)}{2 \varepsilon_{0}^{\prime}}\left(\varepsilon_{1}(-q)-\frac{\delta \varepsilon_{0}^{\prime}}{T}\right)^{2}+\frac{\pi^{2} T^{2} f(-q)}{6 \varepsilon_{0}^{\prime}}+\text { h.o.c. . }
$$

Combining (A.6), (A.7) with (A.3) we obtain after simple algebra

$$
\begin{aligned}
J[\varepsilon]=-\int_{-q}^{q} f(\lambda) \varepsilon(\lambda) d \lambda+\frac{T^{2} \pi^{2}}{6 \varepsilon_{0}^{\prime}}(f(q)+f(-q)) & \\
& +\frac{T^{2} f(q) \varepsilon_{1}^{2}(q)}{2 \varepsilon_{0}^{\prime}}+\frac{T^{2} f(-q) \varepsilon_{1}^{2}(-q)}{2 \varepsilon_{0}^{\prime}}+\text { h.o.c. . }
\end{aligned}
$$

In a similar way, one can obtain the low-temperature expansion of integrals involving the function $u(\lambda)$

$$
J[u]=T \int_{\mathbb{R}} f(\lambda) \log \left(1+e^{-\frac{u(\lambda)}{T}}\right) d \lambda .
$$

Since $u_{0}(\lambda)=\varepsilon_{0}(\lambda)$, exactly the same considerations lead us to the estimate

$$
\begin{aligned}
J[u]=-\int_{-q}^{q} f(\lambda) u(\lambda) d \lambda+\frac{T^{2} \pi^{2}}{6 \varepsilon_{0}^{\prime}}(f(q)+ & f(-q)) \\
& +\frac{T^{2} f(q) u_{1}^{2}(q)}{2 \varepsilon_{0}^{\prime}}+\frac{T^{2} f(-q) u_{1}^{2}(-q)}{2 \varepsilon_{0}^{\prime}}+O\left(T^{3}\right) .
\end{aligned}
$$




\section{B Estimates of integrals with singular functions}

\section{B.1 The Cauchy transform in the vicinities of $\pm q$}

In this section we determine the leading $T \rightarrow 0$ behavior of $L_{\mathbb{R}}[z](\lambda)$. Its depends on where $\lambda$ is located. Recall that

$$
L_{\mathbb{R}}[z](\lambda)=\frac{-1}{2 \pi i} \int_{\mathbb{R}} \log \left(\frac{1+e^{-\frac{u(\mu)}{T}}}{1+e^{-\frac{\varepsilon(\mu)}{T}}}\right) \frac{d \mu}{\mu-\lambda} .
$$

If $\lambda$ is separated from $\pm q$, then obviously

$$
\lim _{T \rightarrow 0} L_{\mathbb{R}}[z](\lambda)=\frac{1}{2 \pi i} \int_{-q}^{q} \frac{u_{1}(\mu) d \mu}{\mu-\lambda}=\frac{1}{2 \pi i} L_{[-q, q]}\left[u_{1}\right](\lambda),
$$

where $u_{1}(\lambda)$ is given by (3.22).

Let now $\lambda \rightarrow q$ as $T \rightarrow 0$. We denote $\lambda=\lambda_{ \pm}$, if $\lambda$ approaches $q$ from the upper (resp. lower) half-plane. Let again $\delta>0$ be such that $\delta \rightarrow 0$ as $T \rightarrow 0$, while $\delta / T \rightarrow \infty$ in the $T \rightarrow 0$ limit. Consider the contributions to the integral (B.1) coming from different intervals of integration. Obviously, when $T \rightarrow 0$ the integrals over domains $\lambda>q+\delta$ and $\lambda<-q-\delta$ produce exponentially small corrections. On the other hand $z(\lambda)$ can be approximated by $\frac{u_{1}(\lambda)}{2 \pi i}$ on the interval $[-q-\delta, q-\delta]$ :

$$
\frac{-1}{2 \pi i} \int_{-q-\delta}^{q-\delta} \log \left(\frac{1+e^{-\frac{u(\mu)}{T}}}{1+e^{-\frac{\varepsilon(\mu)}{T}}}\right) \frac{d \mu}{\mu-\lambda_{ \pm}} \hookrightarrow \frac{1}{2 \pi i} \int_{-q-\delta}^{q-\delta} \frac{u_{1}(\mu) d \mu}{\mu-\lambda_{ \pm}}, \quad T \rightarrow 0 .
$$

Extracting the divergent part we obtain

$$
\frac{1}{2 \pi i} \int_{-q-\delta}^{q-\delta} \frac{u_{1}(\mu) d \mu}{\mu-\lambda_{ \pm}} \rightarrow \frac{1}{2 \pi i} \int_{-q}^{q} \frac{u_{1}(\mu)-u_{1}}{\mu-\lambda_{ \pm}} d \mu+\frac{u_{1}}{2 \pi i} \log \left(\frac{\lambda_{ \pm}-q+\delta}{\lambda_{ \pm}+q}\right), \quad \delta \rightarrow 0,
$$

and we remind that $u_{1}=u_{1}( \pm q)$.

It remains to compute the integral over $[q-\delta, q+\delta]$. Following the method of the previous section we linearize the functions $u(\mu)$ and $\varepsilon(\mu)$ in the vicinity of $\mu=q$. Then we have

$$
I_{q} \equiv \frac{-1}{2 \pi i} \int_{q-\delta}^{q+\delta} \log \left(\frac{1+e^{-\frac{u(\mu)}{T}}}{1+e^{-\frac{\varepsilon(\mu)}{T}}}\right) \frac{d \mu}{\mu-\lambda_{ \pm}} \hookrightarrow \frac{-1}{2 \pi i} \int_{-\delta}^{\delta} \log \left(\frac{1+e^{-\frac{\mu \varepsilon_{0}^{\prime}}{T}-u_{1}}}{1+e^{-\frac{\mu \varepsilon_{0}^{\prime}}{T}}}\right) \frac{d \mu}{\mu-\left(\lambda_{ \pm}-q\right)} .
$$

Replacing $\mu \varepsilon_{0}^{\prime} / T=\xi$ we arrive at

$$
I_{q}=\frac{-1}{2 \pi i} \int_{-\delta \varepsilon_{0}^{\prime} / T}^{\delta \varepsilon_{0}^{\prime} / T}\left[\log \left(\frac{e^{\xi}+e^{-u_{1}}}{e^{\xi}+1}\right)+u_{1} \Theta(-\xi-c)\right] \frac{d \xi}{\xi-t_{ \pm}}+\frac{u_{1}}{2 \pi i} \log \left(\frac{t_{ \pm}+c}{t_{ \pm}+\frac{\delta \varepsilon_{0}^{\prime}}{T}}\right)
$$


where $c$ is an arbitrary positive constant and we have set $t_{ \pm}=\left(\lambda_{ \pm}-q\right) \varepsilon_{0}^{\prime} / T$. We can send now $\delta / T \rightarrow \infty$. Substituting into (B.6)

$$
\frac{1}{\xi-t_{ \pm}}= \pm i \int_{0}^{\infty} e^{\mp i \omega\left(\xi-t_{ \pm}\right)} d \omega
$$

we arrive at

$$
\begin{array}{r}
I_{q}=\frac{\mp 1}{2 \pi} \int_{-\infty}^{\infty} d \xi \int_{0}^{\infty} d \omega\left[\log \left(\frac{e^{\xi}+e^{-u_{1}}}{e^{\xi}+1}\right)+u_{1} \Theta(-\xi-c)\right] e^{\mp i \omega\left(\xi-t_{ \pm}\right)} \\
+\frac{u_{1}}{2 \pi i} \log \left(\frac{t_{ \pm}+c}{t_{ \pm}+\frac{\delta \varepsilon_{0}^{\prime}}{T}}\right) .
\end{array}
$$

The integral over $\xi$ can be calculated by means of an integration by parts followed by a computation of the residues at $e^{\xi}+e^{-u_{1}}=0$ and $e^{\xi}+1=0$ :

$$
\frac{1}{2 \pi} \int_{-\infty}^{\infty} d \xi\left[\log \left(\frac{e^{\xi}+e^{-u_{1}}}{e^{\xi}+1}\right)+u_{1} \Theta(-\xi-c)\right] e^{\mp i \omega \xi}=\frac{1-e^{ \pm i \omega u_{1}}}{2 \omega \sinh (\pi \omega)} \mp \frac{u_{1} e^{ \pm i \omega c}}{2 \pi i \omega} .
$$

Thus, we arrive at

$$
I_{q}=\frac{u_{1}}{2 \pi i} \log \left(\frac{t_{ \pm}+c}{t_{ \pm}+\frac{\delta \varepsilon_{0}^{\prime}}{T}}\right) \pm \frac{1}{2 \pi} \int_{0}^{\infty} \frac{d \omega}{\omega}\left[\mp i u_{1} e^{ \pm i \omega c}-\frac{\pi}{\sinh (\pi \omega)}\left(1-e^{ \pm i \omega u_{1}}\right)\right] e^{ \pm i \omega t_{ \pm}} .
$$

Due to (3.26) the last integral is convergent. It can be computed in terms of the $\Gamma$-functions via

$$
\int_{0}^{\infty} \frac{e^{-p \omega} d \omega}{\omega}\left[b-a-\frac{\pi}{\sinh (\pi \omega)}\left(e^{-a \omega}-e^{-b \omega}\right)\right]=(a-b) \log \left(\frac{p}{2 \pi}\right)+2 \pi \log \frac{\Gamma\left(\frac{p+b}{2 \pi}+\frac{1}{2}\right)}{\Gamma\left(\frac{p+a}{2 \pi}+\frac{1}{2}\right)} .
$$

Thus, we obtain

$$
I_{q}=\frac{u_{1}}{2 \pi i} \log \left(\frac{ \pm 2 \pi i T}{\left(\lambda_{ \pm}-q+\delta\right) \varepsilon_{0}^{\prime}}\right) \pm \log \frac{\Gamma\left(\frac{1}{2} \pm \frac{\left(\lambda_{ \pm}-q\right) \varepsilon_{0}^{\prime}}{2 \pi i T} \pm \frac{u_{1}}{2 \pi i}\right)}{\Gamma\left(\frac{1}{2} \pm \frac{\left(\lambda_{ \pm}-q\right) \varepsilon_{0}^{\prime}}{2 \pi i T}\right)} .
$$

Combining this result with (B.4) we find the following estimate

$$
\begin{aligned}
L_{\mathbb{R}}[z]\left(\lambda_{ \pm}\right) & =\frac{1}{2 \pi i} \int_{-q}^{q} \frac{u_{1}(\mu)-u_{1}}{\mu-\lambda_{ \pm}} d \mu+\frac{u_{1}}{2 \pi i} \log \left(\frac{\lambda_{ \pm}-q}{\lambda_{ \pm}+q}\right) \\
& -\frac{u_{1}}{2 \pi i} \log \left(\frac{\left(\lambda_{ \pm}-q\right) \varepsilon_{0}^{\prime}}{ \pm 2 \pi i T}\right) \pm \log \frac{\Gamma\left(\frac{1}{2} \pm \frac{\left(\lambda_{ \pm}-q\right) \varepsilon_{0}^{\prime}}{2 \pi i T} \pm \frac{u_{1}}{2 \pi i}\right)}{\Gamma\left(\frac{1}{2} \pm \frac{\left(\lambda_{ \pm}-q\right) \varepsilon_{0}^{\prime}}{2 \pi i T}\right)}, \quad T \rightarrow 0, \quad \lambda \sim q .
\end{aligned}
$$


Similarly, if $\lambda \rightarrow-q$ as $T \rightarrow 0$ one has

$$
\begin{aligned}
L_{\mathbb{R}}[z]\left(\lambda_{ \pm}\right) & =\frac{1}{2 \pi i} \int_{-q}^{q} \frac{u_{1}(\mu)-u_{1}}{\mu-\lambda_{ \pm}} d \mu+\frac{u_{1}}{2 \pi i} \log \left(\frac{\lambda_{ \pm}-q}{\lambda_{ \pm}+q}\right) \\
& +\frac{u_{1}}{2 \pi i} \log \left(\frac{\left(\lambda_{ \pm}+q\right) \varepsilon_{0}^{\prime}}{ \pm 2 \pi i T}\right) \pm \log \frac{\Gamma\left(\frac{1}{2} \pm \frac{\left(\lambda_{ \pm}+q\right) \varepsilon_{0}^{\prime}}{2 \pi i T} \mp \frac{u_{1}}{2 \pi i}\right)}{\Gamma\left(\frac{1}{2} \pm \frac{\left(\lambda_{ \pm}+q\right) \varepsilon_{0}^{\prime}}{2 \pi i T}\right)}, \quad T \rightarrow 0, \quad \lambda \sim-q .
\end{aligned}
$$

\section{B.2 The double integral}

Consider now the low temperature behavior of the integral A given in (5.17). As usual we split the integration domain into several pieces $A=A_{-}+A_{-q}+A_{0}+A_{q}+A_{+}$. The integral $A_{+}$(resp. $A_{-}$) over the domain $\lambda>q+\delta$ (resp. $\lambda<-q-\delta$ ) are again exponentially small in respect to the $T \rightarrow 0$ limit. When $\lambda \in[-q-\delta, q-\delta]$, we can use the expression (B.2) for $L_{\mathbb{R}}[z]\left(\lambda_{+}\right)$and also replace $z(\lambda)$ by $\frac{u_{1}(\lambda)}{2 \pi i}$. This gives

$$
A_{0} \equiv \frac{1}{(2 \pi i)^{2}} \int_{-q+\delta}^{q-\delta} d \lambda u_{1}(\lambda) \partial_{\lambda} \int_{-q}^{q} d \mu \frac{u_{1}(\mu)}{\mu-\lambda_{+}}
$$

Integrating by parts we arrive at

$$
A_{0}=\frac{-1}{(2 \pi i)^{2}} \int_{-q+\delta}^{q-\delta} d \lambda \int_{-q}^{q} d \mu \frac{u_{1}^{\prime}(\lambda) u_{1}(\mu)}{\mu-\lambda_{+}}+\frac{u_{1}(q-\delta)}{(2 \pi i)^{2}}\left(\int_{-q}^{q} \frac{u_{1}(\mu) d \mu}{\mu_{-}-q+\delta}-\int_{-q}^{q} \frac{u_{1}(\mu) d \mu}{\mu_{-}+q-\delta}\right) .
$$

Here we have used that $u_{1}(\lambda)=u_{1}(-\lambda)$. This last property also allows one to symmetrize the integrand, so that upon sending $\delta \rightarrow 0$, we get

$$
\frac{-1}{(2 \pi i)^{2}} \int_{-q+\delta}^{q-\delta} d \lambda \int_{-q}^{q} d \mu \frac{u_{1}^{\prime}(\lambda) u_{1}(\mu)}{\mu-\lambda_{+}}=\frac{1}{2(2 \pi i)^{2}} \int_{-q}^{q} \frac{u_{1}^{\prime}(\lambda) u_{1}(\mu)-u_{1}(\lambda) u_{1}^{\prime}(\mu)}{\lambda-\mu} d \lambda d \mu, \quad \delta \rightarrow 0 .
$$

Extracting then the divergent part from the single integrals in (B.16) we find

$$
A_{0} \rightarrow C_{1}\left[\frac{u_{1}(\lambda)}{2 \pi i}\right]+\frac{2 u_{1}^{2}}{(2 \pi i)^{2}} \log \left(\frac{\delta}{2 q}\right), \quad \delta \rightarrow 0
$$

where the functional $C_{1}[F]$ is defined in (5.23).

Consider now the contribution $A_{q}$ coming from the interval $q-\delta<\lambda<q+\delta$ :

$$
A_{q}=\int_{q-\delta}^{q+\delta}\left(\partial_{\lambda} L_{\mathbb{R}}[z]\left(\lambda_{+}\right)\right) \cdot z(\lambda) d \lambda
$$


Substituting here $(\underline{\mathrm{B} .13})$ we arrive at $A_{q}=A_{q}^{(1)}+A_{q}^{(2)}+A_{q}^{(3)}$, where

$$
\begin{gathered}
A_{q}^{(1)}=\frac{1}{2 \pi i} \int_{q-\delta}^{q+\delta} z(\lambda) \partial_{\lambda}\left[\int_{-q}^{q} \frac{u_{1}(\mu)-u_{1}}{\mu-\lambda_{ \pm}} d \mu-\frac{u_{1}}{2 \pi i} \log \left(\lambda_{ \pm}+q\right)\right] d \lambda, \\
A_{q}^{(2)}=\frac{-u_{1}}{(2 \pi i)^{2}} \int_{-q-\delta}^{q+\delta} \log \left(\frac{1+e^{-\frac{u(\lambda)}{T}}}{1+e^{-\frac{\varepsilon(\lambda)}{T}}}\right) \frac{d \lambda}{\lambda_{+}-q},
\end{gathered}
$$

and

$$
\begin{array}{r}
A_{q}^{(3)}=\frac{-1}{2 \pi i} \int_{-q-\delta}^{q+\delta} \partial_{\lambda}\left\{\log \frac{\Gamma\left(\frac{1}{2}+\frac{\left(\lambda_{+}-q\right) \varepsilon_{0}^{\prime}}{2 \pi i T}+\frac{u_{1}}{2 \pi i}\right)}{\Gamma\left(\frac{1}{2}+\frac{\left(\lambda_{+}-q\right) \varepsilon_{0}^{\prime}}{2 \pi i T}\right)}-\frac{u_{1}}{2 \pi i} \log \left(\frac{\left(\lambda_{+}-q\right) \varepsilon_{0}^{\prime}}{2 \pi i T}\right)\right\} \\
\times \log \left(\frac{1+e^{-\frac{u(\lambda)}{T}}}{1+e^{-\frac{\varepsilon(\lambda)}{T}}}\right) d \lambda .
\end{array}
$$

It is easy to see that $A_{q}^{(1)} \rightarrow 0$ as $\delta \rightarrow 0$, because the integrand is a bounded function as $T \rightarrow 0$. The integral $A_{q}^{(2)}$ can be estimated similarly to (B.5):

$$
A_{q}^{(2)}=\frac{u_{1}^{2}}{(2 \pi i)^{2}} \log \left(\frac{-2 \pi i T}{\delta \varepsilon_{0}^{\prime}}\right)-\frac{u_{1}}{2 \pi i} \log \frac{\Gamma\left(\frac{1}{2}-\frac{u_{1}}{2 \pi i}\right)}{\Gamma\left(\frac{1}{2}\right)}, \quad T \rightarrow 0, \quad \delta \rightarrow 0 .
$$

As for the remaining integral $A_{q}^{(3)}$, its leading behavior is obtained by a linearization of the functions $u(\lambda)$ and $\varepsilon(\lambda)$ in the vicinity of $\lambda=q$. After the change of variables $\xi=(\lambda-q) \varepsilon_{0}^{\prime} / T$ followed by an integration by parts, we find in the $\delta \rightarrow 0, T \rightarrow 0$ limit

$$
A_{q}^{(3)}=\frac{1}{2 \pi i} \int_{-\infty}^{\infty}\left\{\log \frac{\Gamma\left(\frac{1}{2}+\frac{\xi+u_{1}}{2 \pi i}\right)}{\Gamma\left(\frac{1}{2}+\frac{\xi}{2 \pi i}\right)}-\frac{u_{1}}{2 \pi i} \log \left(\frac{\xi+i 0}{2 \pi i}\right)\right\}\left(\frac{1}{1+e^{-\xi-u_{1}}}-\frac{1}{1+e^{-\xi}}\right) d \xi
$$

We close the integration contour in the upper half-plane and compute the integral (B.24) by residues. These are located at $\xi=-u_{1}+\pi i(2 k+1)$ and $\xi=\pi i(2 k+1), k=0,1 \ldots$ Hence,

$$
\begin{aligned}
& A_{q}^{(3)}=\sum_{k=1}^{\infty} {\left[\log \frac{\Gamma^{2}(k)}{\Gamma\left(k+\frac{u_{1}}{2 \pi i}\right) \Gamma\left(k-\frac{u_{1}}{2 \pi i}\right)}-\frac{u_{1}}{2 \pi i} \log \left(\frac{\left.k-\frac{1}{2}-\frac{u_{1}}{2 \pi i}\right)}{k-\frac{1}{2}}\right)\right] } \\
&=\log G\left(1, \frac{u_{1}}{2 \pi i}\right)+\frac{u_{1}}{2 \pi i} \log \frac{\Gamma\left(\frac{1}{2}-\frac{u_{1}}{2 \pi i}\right)}{\Gamma\left(\frac{1}{2}\right)} \\
& \quad+\lim _{N \rightarrow \infty}\left[\log \frac{G^{2}(N+1)}{G\left(N+1+\frac{u_{1}}{2 \pi i}\right) G\left(N+1-\frac{u_{1}}{2 \pi i}\right)}-\frac{u_{1}}{2 \pi i} \log \frac{\Gamma\left(N+\frac{1}{2}-\frac{u_{1}}{2 \pi i}\right)}{\Gamma\left(N+\frac{1}{2}\right)}\right],
\end{aligned}
$$


where $G(x)$ is the Barnes function and $G(1, x)=G(1+x) G(1-x)$. Using the asymptotic behavior of the $\Gamma$ and Barnes functions for $z \rightarrow \infty$ with $z \notin \mathbb{R}_{-}$

$$
\begin{aligned}
& \log G(z+1+a)-\log G(z+1)=a \log \sqrt{2 \pi}+\frac{a}{2}(2 z+a) \log z-a z+\mathrm{o}(1), \\
& \log \Gamma(z+1+a)-\log \Gamma(z+1)=a \log z+\mathrm{o}(1),
\end{aligned}
$$

we find that the limit in the last line of (B.25) vanishes. Hence,

$$
A_{q}^{(3)}=\log G\left(1, \frac{u_{1}}{2 \pi i}\right)+\frac{u_{1}}{2 \pi i} \log \frac{\Gamma\left(\frac{1}{2}-\frac{u_{1}}{2 \pi i}\right)}{\Gamma\left(\frac{1}{2}\right)} .
$$

Combining this result with (B.23) we find

$$
A_{q} \rightarrow \frac{u_{1}^{2}}{(2 \pi i)^{2}} \log \left(\frac{-2 \pi i T}{\delta \varepsilon_{0}^{\prime}}\right)+\log G\left(1, \frac{u_{1}}{2 \pi i}\right), \quad T \rightarrow 0, \quad \delta \rightarrow 0 .
$$

Similar calculation in the vicinity of the point $-q$ leads us to the following below contribution coming from the interval $q-\delta<\lambda<q+\delta$ :

$$
A_{-q} \equiv \int_{-q-\delta}^{-q+\delta}\left(\partial_{\lambda} L_{\mathbb{R}}[z]\left(\lambda_{+}\right)\right) \cdot z(\lambda) d \lambda \rightarrow \frac{u_{1}^{2}}{(2 \pi i)^{2}} \log \left(\frac{2 \pi i T}{\delta \varepsilon_{0}^{\prime}}\right)+\log G\left(1, \frac{u_{1}}{2 \pi i}\right), \quad T \rightarrow 0, \quad \delta \rightarrow 0 .
$$

Thus, taking into account (B.27), (B.28) and (B.18) we finally obtain

$$
A \rightarrow C_{1}\left[\frac{u_{1}(\lambda)}{2 \pi i}\right]-2\left(\frac{u_{1}}{2 \pi i}\right)^{2} \log \left(\frac{q \varepsilon_{0}^{\prime}}{\pi T}\right)+2 \log G\left(1, \frac{u_{1}}{2 \pi i}\right), \quad T \rightarrow 0 .
$$

\section{Smooth part of the amplitude}

In this section we give the exact expression for the smooth part of the amplitude $B_{s}^{(0)}\left[\alpha_{\ell} Z\right]=$ $\lim _{T \rightarrow 0} B_{s}[u]$. Provided the condition (3.12) holds, we have

$$
\begin{aligned}
& B_{s}^{(0)}\left[\alpha_{\ell} Z\right]=\left(e^{2 \pi i \alpha}-1\right)^{2} e^{-C_{0}} \frac{\operatorname{det}\left(I+\frac{1}{2 \pi i} \hat{U}^{(1)}\left[\alpha_{\ell} Z\right]\right) \operatorname{det}\left(I+\frac{1}{2 \pi i} \hat{U}^{(2)}\left[\alpha_{\ell} Z\right]\right)}{\left(\operatorname{det}\left[I-\frac{1}{2 \pi} K\right]\right)^{2}} \\
& \quad \times\left(e^{-\alpha_{\ell} L[Z]\left(\theta_{1}+i c\right)}-e^{2 \pi i \alpha-\alpha_{\ell} L[Z]\left(\theta_{1}-i c\right)}\right)^{-1}\left(e^{\alpha_{\ell} L[Z]\left(\theta_{2}-i c\right)}-e^{2 \pi i \alpha+\alpha_{\ell} L[Z]\left(\theta_{2}+i c\right)}\right)^{-1} .
\end{aligned}
$$

Here $L[Z](\omega)$ stands the Cauchy transform of the dressed charge $Z(\lambda)$ on the interval $[-q, q]$, and $C_{0}$ is given by (5.6). The integral operator $I-\frac{1}{2 \pi} K$ acts on the interval $[-q, q]$ and its kernel was defined by (2.3). The operators $I+\frac{1}{2 \pi i} \hat{U}^{(1)}\left[\alpha_{\ell} Z\right]$ and $I+\frac{1}{2 \pi i} \hat{U}^{(2)}\left[\alpha_{\ell} Z\right]$ act on a anticlockwise oriented closed contour surrounding $[-q, q]$. Their kernels are

$$
\hat{U}^{(1)}\left(w, w^{\prime},\left[\alpha_{\ell} Z\right]\right)=-e^{-\alpha_{\ell} L[Z](w)} \cdot \frac{K_{\alpha}\left(w-w^{\prime}\right)-K_{\alpha}\left(\theta_{1}-w^{\prime}\right)}{e^{-\alpha_{\ell} L[Z](w+i c)}-e^{2 \pi i \alpha-\alpha_{\ell} L[Z](w-i c)}},
$$


and

$$
\hat{U}^{(2)}\left(w, w^{\prime},\left[\alpha_{\ell} Z\right]\right)=e^{\alpha_{\ell} L[Z]\left(w^{\prime}\right)} \cdot \frac{K_{\alpha}\left(w-w^{\prime}\right)-K_{\alpha}\left(w-\theta_{2}\right)}{e^{\alpha_{\ell} L[Z]\left(w^{\prime}-i c\right)}-e^{2 \pi i \alpha+\alpha_{\ell} L[Z]\left(w^{\prime}+i c\right)}},
$$

where $K_{\alpha}(\lambda)$ is given by (2.18). Finally parameters $\theta_{1}$ and $\theta_{2}$ are arbitrary complex numbers lying inside of the contour where the operators $\hat{U}^{(1,2)}\left(w, w^{\prime},\left[\alpha_{\ell} Z\right]\right)$ act. If we set $\theta_{1}=-q$ and $\theta_{2}=q$, then we reproduce the smooth part of form factors of the $\mathbf{P}_{\ell}$ class obtained [17].

\section{References}

[1] K. K. Kozlowski, J. M. Maillet, N. A. Slavnov, Long-distance behavior of temperature correlation functions in the one-dimensional Bose-gas, math-ph: 10113149.

[2] A. Seel, T. Bhattacharyya, F. Göhmann, A. Klümper, Journ. Stat. Mech. (2007) P08030.

[3] A. Klümper and M. Batchelor, J. Phys. A: Math. Gen. 23 (1990) L189.

[4] A. Klümper, M. Batchelor and P. Pearce, J. Phys. A: Math. Gen. 24 (1991) 3111.

[5] A. Klümper, Z. Phys. B91 (1993) 507.

[6] A. Klümper, T. Wehner and J. Zittartz, J. Phys. A : Math. Gen. 26 (1993) 2815.

[7] A. Klümper, Lect. Notes in Physics, 645 (2004) 349.

[8] F. D. M. Haldane, Phys. Rev. Lett. 47, (1981) 1840

[9] A. A. Belavin, A.M. Polyakov and A.B. Zamolodchikov, Nucl. Phys. B241 (1984) 333.

[10] J. L. Cardy, J. Phys. A: Math. Gen. 17 (1984) L385.

[11] H. J. de Vega and F. Woynarovich, Nucl. Phys. B251 (1985) 439.

[12] J. L. Cardy, Nucl. Phys. B270 (1986) 186.

[13] H. W. Blöte, J. L. Cardy and M. P. Nightingale, Phys. Rev. Lett. 56 (1986) 742.

[14] I. Affleck, Phys. Rev. Lett. 56 (1986) 745.

[15] N. Bogolyubov, A. Izergin and N. Reshetikhin, Zh. Eksp. Theor. Fiz. 44 (1986) 405.

[16] J. L. Cardy, "Scaling and renormalization in statistical physics", Cambridge Lecture notes in Physics, 1996.

[17] N. Kitanine, K. K. Kozlowski, J. M. Maillet, N. A. Slavnov and V. Terras, Thermodynamic limit of particle-hole form factors in the massless XXZ Heisenberg chain, arXiv:Math-ph: 1003.4557. 
[18] C. N. Yang and C. P. Yang, J. Math. Phys., 10 (1969) 1115.

[19] N. Kitanine, K. K. Kozlowski, J. M. Maillet, N. A. Slavnov and V. Terras, J. Stat. Mech. 042009 (2009) P04003.

[20] J.D. Johnson and B.M. McCoy, Phys. Rev. A6 (1972) 1613

[21] L. Mezincescu and R.I. Nepomechie, in Quantum Groups, Integrable Models and Statistical Systems, ed. by J. LeTourneux and L. Vinet, World Scientific Publishing Co., (1993) 168; arXiv:hep-th/9212124v1.

[22] N.A. Slavnov, Theor. Math. Phys., 121 (1999) 1358.

[23] N. Kitanine, K. K. Kozlowski, J. M. Maillet, N. A. Slavnov, and V. Terras, J. Stat. Mech. Theory Exp. (2007) P01022.

[24] A. Berkovich and G. Murthy, J. Phys. A: Math. Gen. 21 (1988) L395.

[25] A. Berkovich and G. Murthy, J. Phys. A: Math. Gen. 21 (1988) 3703.

[26] E. H. Lieb and W. Liniger, Phys. Rev. 130 (1963) 1605.

[27] E. H. Lieb, Phys. Rev. 130 (1963) 1616.

[28] E. H. Lieb and D. C. Mattis, Mathematical Physics in One Dimension, Academic Press, New-York, 1966.

[29] V. E. Korepin, N. M. Bogoliubov, A. G. Izergin, Quantum Inverse Scattering Method and Correlation Functions, Cambridge University Press, 1993.

[30] N.A. Slavnov, Theor. Math. Phys. 165 (2010) 1262.

[31] N. Kitanine, K. K. Kozlowski, J. M. Maillet, N. A. Slavnov and V. Terras, Form factor approach to the long-distance asymptotic behavior of correlation functions in critical models, to appear. 